\title{
Novel formulations and modeling enhancements for the dynamic berth allocation problem
}

\author{
Arthur Kramer ${ }^{\mathrm{a}}$, Eduardo Lalla-Ruiz ${ }^{\mathrm{b}, *}$, Manuel Iori ${ }^{\mathrm{a}}$, Stefan Voß ${ }^{\mathrm{c}, \mathrm{d}}$ \\ a Department of Sciences and Methods for Engineering, University of Modena and Reggio Emilia Via Amendola 2, Pad. Morselli, Reggio Emilia, 42122 Italy \\ ${ }^{\mathrm{b}}$ Department of Industrial Engineering and Business Information Systems, University of Twente P.O. Box 217, AE Enschede, 7500 the Netherlands \\ ' Institute of Information Systems, University of Hamburg Von-Melle-Park 5, Room 306520146 Hamburg, Germany \\ ${ }^{\mathrm{d}}$ Escuela de Ingenieriá Industrial, Pontificia Universidad Católica de Valparaíso, Valparaíso, Chile
}

\section{A R T I C L E I N F O}

\section{Article history:}

Received 11 September 2018

Accepted 21 March 2019

Available online 28 March 2019

\section{Keywords:}

OR in maritime industry

Dynamic berth allocation problem

Novel formulations

Modeling enhancements

\begin{abstract}
A B S T R A C T
This paper addresses the well-known dynamic berth allocation problem (DBAP), which finds numerous applications at container terminals aiming to allocate and schedule incoming container vessels into berthing positions along the quay. Due to its impact on ports' performance, having efficient DBAP formulations is of great importance, especially for determining optimal schedules in quick time as well as aiding managers and developers in the assessment of solution strategies and approximate approaches. In this work, we propose two novel formulations, a time-indexed formulation and an arc-flow one, to efficiently tackle the DBAP. Additionally, to improve computational performance, we propose problem-based modeling enhancements and a variable-fixing procedure that allows to discard some variables by considering their reduced costs. By means of these contributions, we improve the models' performance for those instances where the optimal solutions were already known, and we solve to optimality for the first time other instances from the literature.
\end{abstract}

(c) 2019 Elsevier B.V. All rights reserved.

\section{Introduction}

The management of limited resources at maritime container terminals has a direct and relevant impact on their productivity and competitiveness. This holds, especially, in those cases where for geographical or monetary reasons the terminals are forced to find different ways to expand their capacity. Thus, terminal managers require the use of suitable methods and approaches to efficiently exploit resources at maritime terminals. This involves the need for reliable and fast approaches for providing schedules within reasonable computational times, as well as having efficient mathematical models enabling the proper evaluation of those schedules by means of a given objective function. As indicated by Notteboom (2006), over 90\% of the delayed vessel schedules are due to port access and terminal operations that, as pointed out by Steenken, Voß, and Stahlbock (2004), directly involve the management of berths. In this regard, berth planning is a relevant process within terminal operating systems (Heilig \& Voß, 2018) or within information platforms (e.g., Choi, Kim, Park, Park, \& Lee, 2003). The necessity to solve such process responds to a real-world daily

\footnotetext{
* Corresponding author.
}

E-mail addresses: arthur.kramer@unimore.it (A. Kramer), e.a.lalla@utwente.nl (E. Lalla-Ruiz), manuel.iori@unimore.it (M. Iori), stefan.voss@uni-hamburg.de (S. Voß). necessity to use terminal resources as best as possible. Hence, it becomes essential for terminal operators and related practitioners to rely on efficient solution approaches in order to suitably manage the use of those limited and impacting resources such as berths.

The above issue leads to the definition of the well-known berth allocation problem (BAP), which seeks to assign and schedule incoming vessels arriving at the terminal into berthing positions with the aim of optimizing a given objective function (e.g. minimize the waiting time of the vessels, maximum departure time, etc.). In this way, optimal berthing positions and times for all vessels are provided, allowing planned berthing instructions while efficiently using the quay space. Different variants of the BAP have been proposed (see e.g. Bierwirth \& Meisel 2010; 2015). Among them, the most referenced and known one is the dynamic berth allocation problem (DBAP, Cordeau, Laporte, Legato, \& Moccia 2005; Imai, Nishimura, \& Papadimitriou 2001). The DBAP aims at allocating container vessels along the quay partitioned into berths while reducing the sum of vessels turnaround time. In contrast to the static case (where all the vessels are at the port at the beginning of the planning horizon), the DBAP considers that vessels arrive along the planning horizon, i.e., the term "dynamic" means that the vessels arrive at different times of the day; nevertheless, all problem information is known in advance. Due to the difficulty of this problem, decision support approaches are necessary to provide proper solutions. This opens up the discussion on how and from 
which standpoint algorithmic techniques can contribute to enhancing the management of berthing resources in port environments. Based on the literature (e.g. Bierwirth \& Meisel 2010; Lalla-Ruiz 2016; Stahlbock \& Voß 2008) different ways for solving the DBAP can be indicated:

1. Heuristic and metaheuristic approaches. They allow decision makers to provide feasible solutions within reasonable computational times. The application of these methods is suggested in situations where fast solutions are requested such as in dynamic environments requiring replannning or in integrative and rich solution systems where the solution of a problem is an input for other problems. Some examples applied to the DBAP can be consulted in Cordeau, Gaudioso, Laporte, and Moccia (2007), De Oliveira, Mauri, and Lorena (2012), Lalla-Ruiz, Melián-Batista, and Moreno-Vega (2012), Ting, Wu, and Chou (2014), Şahin and Kuvvetli (2016).

2. Matheuristic approaches. They integrate both exact and metaheuristic techniques, in such a way that some of the capabilities of those methods can be jointly exploited. The integration of exact approaches within a metaheuristic or viceversa can lead to higher computational times than heuristic methods, but may also lead to a better performance robustness and quality of the solutions in some applications. For approaches in this regard, the reader is referred to Lalla-Ruiz and Voß (2016b), Nishi, Okura, Lalla-Ruiz, and Voß (2016).

3. Exact approaches. The main advantage of this type of approaches is to seek optimality by means of bounds at the cost of requiring important computational efforts as the size of the instance increases. Counting on well-defined and assessed mathematical formulations, as well as exact algorithms, permits researchers and practitioners to evaluate the performance of their heuristic and matheuristic methods, while increasing the expertise and knowledge on the given problem and technique. For instance, a review of current formulations can be found in Buhrkal, Zuglian, Ropke, Larsen, and Lusby (2011).

In recent years, the research community has been predominantly proposing heuristic approaches for the DBAP. That provides an incentive to study and develop exact approaches and modeling enhancements in order to aid the evaluation of the heuristics performance. On the other hand, assessing and determining the best formulation considering the evolution of exact solvers permits accelerating the resolution time as well as obtaining additional insights regarding the problem itself. Therefore, in this work, we aim at proposing two different novel ways of modeling the DBAP, i.e., a time-indexed formulation and an arc-flow one. As a follow up of previous studies on this problem, see (Buhrkal et al., 2011), our goal is to provide a detailed comparison between our formulations and the best one proposed in the literature so far, in order to determine their performance and their likely complementarity for tackling the different DBAP benchmark instances. Furthermore, this work also aims at proposing and assessing modeling enhancements and a reduced-cost-based variable-fixing procedure. As discussed below, the results are meaningful as our new formulations enable a relevant time reduction as well as provide optimal solutions not yet reported for several large-size problem instances proposed in the related literature. In addition to that, in order to address more congested scenarios as well as study the performance of the modeling approaches, a new set of large-size problem instances is proposed and investigated.

The remainder of this paper is organized as follows. Section 2 reviews the related literature putting an emphasis on mathematical models. In Section 3, the DBAP is described. Next, the currently best formulations for this problem as well as those proposed in this work are presented in Section 4. Their computational assessment, as well as a detailed comparison, is reported in Section 5. Finally, Section 6 presents the main conclusions of this work and proposes possible future research directions.

\section{Literature works}

The dynamic berth allocation problem (DBAP) was initially proposed by Imai et al. (2001) with the goal of scheduling and allocating vessels along a discrete quay partitioned into berths. Due to its practical and relevant application domain, this problem has attracted a considerable and increasing attention from the research community as one of the most referenced BAP variants as well as from the practitioner side by means of new BAP variants considering DBAP features (e.g., Giallombardo, Moccia, Salani, \& Vacca, 2010; Imai, Yamakawa, \& Huang, 2014; Xu, Li, \& Leung, 2012). Cordeau et al. (2005) reformulated the problem as a multi-depot vehicle routing problem with time-windows (MDVRP-TW) and proposed a tabu search for solving it. In this way, time-window constraints related to contractual agreements between shipping companies and container terminals could be incorporated. Their computational experiments were conducted for a large set of scenarios based on the container terminal of Gioia Tauro (Italy) and the results indicated that the MDVRP-TW formulation was not able to solve small and medium-sized problem instances within the time limit. Christensen and Holst (2008) proposed a generalized set-partitioning problem formulation (GSPP) that is described in detail in Section 4 below. Later, in the work of Buhrkal et al. (2011), all the existing formulations proposed for the DBAP were extensively assessed. The authors indicated that the GSPP formulation clearly outperforms the other formulations in terms of linear bounds and computational time for the problem instances proposed in Cordeau et al. (2005). Nevertheless, (Lalla-Ruiz et al., 2012) studied the GSPP performance on new instances and indicated that, under the computer and general purpose solver version used at that time, the formulation required high-amounts of memory, possibly leading to memory fault problems. Lalla-Ruiz and Voß (2016b) proposed a matheuristic decomposition approach in order to reduce the size of the problems and allowing to tackle them by means of the GSPP formulation. Recently, (Nishi et al., 2016) proposed a new dynamic programming based matheuristic together with new instances to capture congested and larger scenarios. The authors used the GSPP formulation that, thanks to the progress of computers' memory and processors as well as software, allowed to avoid memory problems. Lalla-Ruiz and Voß (2016a) suggested strengthening the optimization model by extracting cuts from redundant constraints.

With regards to approximate approaches, the DBAP has attracted remarkable attention. We focus here on the most recent approaches. De Oliveira et al. (2012) proposed a clustering search with simulated annealing and (Ting et al., 2014) proposed a particle swarm optimization approach. For testing their approaches both works only used the instances provided in Cordeau et al. (2005). Lalla-Ruiz, Melián-Batista, and Moreno-Vega (2016) proposed a cooperative decentralized search and provided a comparison with De Oliveira et al. (2012) and Ting et al. (2014), indicating a relevant time and performance improvement. Mauri, Ribeiro, Lorena, and Laporte (2016) proposed an adaptive large neighborhood search and tested it on all the state-of-the-art instances. All the mentioned metaheuristic approaches reported high-quality solutions in reasonable computational times. Nevertheless, although they were able to provide the optimal solution values for the largest instances proposed by Cordeau et al. (2005), they were not able to evaluate the quality of their approach for the instances of Lalla-Ruiz et al. (2012) as the optimal solutions remained unknown. 


\section{Problem description}

In the DBAP, we are given a set $N=\{1, \ldots, n\}$ of vessels to be allocated within a quay that is divided into a set $M=\{1, \ldots, m\}$ of berths. Each vessel $i \in N$ is available to be served in a given timewindow $\left[t_{i}, t_{i}^{\prime}\right]$, where $t_{i}$ and $t_{i}^{\prime}$ represent its arrival and departure time, respectively. Similarly, each berth $k \in M$ is available to serve vessels in a restricted period $\left[s_{k}, e_{k}\right]$. Furthermore, each vessel $i$ has an associated handling time $\rho_{i k}$ that depends on its assigned berth $k \in M$, and an input priority value $p_{i}$. The objective function of the DBAP is to minimize the total weighted flow time to serve incoming vessels, that is, the time elapsed between the vessels' arrival at the terminal and the completion of their associated operations multiplied by their priority values. Note that once a vessel has started to be served by a berth, its processing cannot be interrupted and restarted afterwards in the same or another berth (i.e., preemption is not allowed).

Fig. 1 presents an example of a DBAP solution. In the figure, a plan for six vessels within three berths is shown. The rectangles represent the vessels and their handling time. Inside each rectangle, we report the service priority of each vessel $\left(p_{i}\right)$. The timewindows of the vessels are represented by the lines at the bottom of the figure. In this case, for example, vessel 1 arrives at time step 3 and should be served until time step 12 . Moreover, the timewindow of each berth is limited by the non-hatched areas. The vessels' handling times are reported in Table 1; those times depend on the assigned berth. Namely, for instance, if vessel 1 is assigned to berth 1 , its handling time would be equal to 7 , which is shorter than the handling time of 8 units that it would have at berth 2 .

As indicated above, the objective value of a DBAP solution is the total weighted service time of the incoming container vessels. In this example, the weighted service times of the six vessels are calculated as follows: vessel $1=(10-3) \cdot(1)=7$, vessel $2=(4-1) \cdot(3)=9$, vessel $3=(6-2) \cdot(6)=24$, vessel $4=$ $(10-4) \cdot(4)=24$, vessel $5=(11-2) \cdot(2)=18$, and vessel $6=$ $(13-11) \cdot(1)=2$. Therefore, the objective function value of this solution is: $7+9+24+24+18+2=84$.

\section{Mathematical formulations for the DBAP}

This section includes the current most efficient mathematical model for the DBAP according to the computational tests in Buhrkal et al. (2011), and the two novel models proposed in this work, namely, the time-indexed formulation and the arc-flow one.

\subsection{Generalized set-partitioning problem formulation}

The generalized set-partitioning problem (GSPP) formulation for the DBAP was proposed by Christensen and Holst (2008). In the GSPP formulation, a column represents a feasible assignment of a vessel to a berth at a certain time. The set of columns is denoted by $\Omega$. Two matrices $A$ and $B$ are defined, both containing $|\Omega|$ columns. Matrix $A=\left(A_{i \omega}\right)$ contains a row for each vessel, and $A_{i \omega}=1$ if and only if column $\omega$ represents an assignment of vessel $i \in N$. Each column of $A$ contains exactly one non-zero element. Matrix $B=\left(B_{p \omega}\right)$ contains a row per (berth, time) position.

The rows of $B$ are indexed by the set $P=\{1,2, \ldots, K\}$ with $K=$ $\sum_{k \in M}\left(e_{k}-s_{k}\right)$. The entry $B_{p \omega}$ is equal to 1 , if and only if, position $p \in P$ is contained in the assignment that column $\omega$ represents. The cost $c_{\omega}$ of any column $\omega \in \Omega$ is the service time of the respective position assignment multiplied by the priority factor $p_{i}$. A binary variable $x_{\omega}$ is equal to 1 if column $\omega$ is used in the solution, and 0 otherwise. With these definitions the GSPP formulation for the
DBAP is stated as follows:

$$
\text { (GSPP) } \min \sum_{w \in \Omega} c_{w} x_{w}
$$

subject to

$\sum_{w \in \Omega} A_{i w} x_{w}=1 \quad i \in N$

$$
\sum_{w \in \Omega} B_{p w} x_{w} \leq 1 \quad p \in P
$$

$x_{w} \in\{0,1\} \quad w \in \Omega$.

The objective function (1) minimizes the total weighted flow time of the vessels. Constraints (2) ensure that all vessels are served. Constraints ( 3 ) guarantee that at a time interval, in a berth, at most one vessel is served. Constraints (4) define the variables' domain. This model contains $\mathcal{O}(n K)$ variables and $\mathcal{O}(n+K)$ constraints.

\subsection{Time-indexed formulation}

The time-indexed (TI) formulation considers the DBAP as an unrelated parallel machine scheduling problem with release dates and deadlines to minimize the total weighted flow time. In addition, it considers machine availability and job-machine incompatibilities. The TI formulation is an adaptation of the one originally proposed by Sousa and Wolsey (1992) for single machine scheduling problems. Let us define $u_{i k}=\min \left\{t_{i}^{\prime}, e_{k}\right\}$ and $l_{i k}=$ $\max \left\{t_{i}, s_{k}\right\}, \forall i \in N, k \in M$. The TI formulation is then:

$$
\text { (TI) } \min \sum_{i \in N} \sum_{k \in M} \sum_{t=l_{i k}}^{u_{i k}-\rho_{i k}} p_{i} x_{i k t}\left(t+\rho_{i k}-t_{i}\right)
$$

subject to

$\sum_{k \in M} \sum_{t=l_{i k}}^{u_{i k}-\rho_{i k}} x_{i k t}=1$

$i \in N$,

$\sum_{i \in N} \sum_{s=\max \left\{t_{i}, s_{k}, t+1-\rho_{i k}\right\}}^{\min \left\{t, t_{i}^{\prime}-\rho_{i k}, e_{k}-\rho_{i k}\right\}} x_{i k s} \leq 1 \quad k \in M, t=s_{k}, \ldots, e_{k}-1$

$x_{i k t} \in\{0,1\}$

$$
i \in N, k \in M, t=l_{i k}, \ldots, u_{i k}-\rho_{i k}
$$

where $x_{i k t}$ is a binary variable taking value 1 if vessel $i$ starts being served at time $t$ by berth $k, 0$ otherwise. The objective function (5) seeks the minimization of the total weighted flow time of the vessels, where the flow time of a vessel $i$ is defined by the difference between its service completion time and its arrival time at the port. Constraints (6) ensure that each vessel is served exactly once. Constraints (7) forbid overlapping among the vessels by imposing that at most 1 vessel is served by a berth at any time. Constraints (8) define the variables' domain. This model contains a pseudo-polynomial number of variables $\mathcal{O}(n K)$ and constraints $\mathcal{O}(n+K)$, a common characteristic of TI formulations.

\subsection{Arc-flow formulation}

Another new way of formulating the DBAP is by means of an arc-flow (AF) formulation. AF models represent problems by using flows on a capacitated network. The main idea is to obtain a oneunit flow from the origin to the sink node for each available resource. In our case, the berths are the resources. For each of them, the origin and the sink node can be seen as $s_{k}$ and $e_{k}$, respectively, and the flow from origin to destination can be interpreted 
Table 1

Example of vessels' handling times and priority values.

\begin{tabular}{lllll}
\hline \multirow{2}{*}{ Vessel } & \multicolumn{2}{l}{ Handling times $\rho_{i k}$} & Priority value $p_{i}$ \\
\cline { 2 - 4 } & Berth 1 & Berth 2 & Berth 3 & \\
\hline 1 & 7 & 8 & 6 & 1 \\
2 & 2 & 3 & 4 & 3 \\
3 & 5 & 5 & 4 & 6 \\
4 & 8 & 6 & 5 & 4 \\
5 & 9 & 8 & 5 & 2 \\
6 & 4 & 2 & 5 & 1 \\
\hline
\end{tabular}

as a sequence of vessels served by the resource $k \in M$. AF formulations have been widely used to formulate different combinatorial optimization problems. In this sense, we address the reader to the works of Valério de Carvalho (1999) and Delorme, Iori, and Martello (2016).

Before introducing the proposed AF formulation, let us define $d_{k t}, \forall k \in M, t=s_{k}, \ldots, e_{k}-1$ as a set of idle variables necessary to allow the presence of idle times between the service of two consecutive vessels. Variable $d_{k t}$ takes value 1 if in the time period from $t$ to $t+1$ the berth $k$ is idle. By using the previous set of variables $x_{i k t}$ and the new variables $d_{k t}$, the DBAP can be formulated as follows:

(AF) $\min \sum_{i \in N} \sum_{k \in M} \sum_{t=l_{i k}}^{u_{i k}-\rho_{i k}} p_{i} x_{i k t}\left(t+\rho_{i k}-t_{i}\right)$

subject to

$\sum_{k \in M} \sum_{t=l_{i k}}^{u_{i k}-\rho_{i k}} x_{i k t}=1 \quad i \in N$,

$\sum_{i \in N} x_{i k t}+d_{k t}-\sum_{i \in N} x_{i, k, t-\rho_{i k}}-d_{k, t-1}=\left\{\begin{array}{ll}1, & \text { if } t=s_{k} \\ -1, & \text { if } t=e_{k} \\ 0, & \text { otherwise }\end{array} \quad \begin{array}{c}k \in M, \\ t=s_{k}, \ldots, e_{k},\end{array}\right.$

$x_{i k t} \in\{0,1\} \quad \begin{gathered}i \in N, k \in M, \\ t=l_{i k}, \ldots, u_{i k}-\rho_{i k},\end{gathered}$

$0 \leq d_{k t} \leq 1 \quad \begin{gathered}k \in M, \\ t=s_{k}, \ldots, e_{k}-1 .\end{gathered}$

The objective function (9) and constraints (10) are equivalent to (5) and (6) in the TI formulation, respectively, whereas constraints (11) impose the flow conservation conditions. Like the TI formulation, the AF formulation, too, is characterized by a pseudo-polynomial number of variables, $\mathcal{O}(n K)$ binary and $\mathcal{O}(K)$ continuous, and constraints $\mathcal{O}(n+K)$. With the aim of illustrating this formulation, Fig. 2 shows the AF solution for the example instance of Fig. 1 and Table 1. From this figure it can be observed that berths 3 and 2 remain idle for one unit of time each at the beginning of their availability periods (modeled by acrs $d_{3,1}$ and $d_{2,0}$, respectively). Vessels 3 and 5 are both served by berth 3 and start (resp. finish) to be served at time 2 and 6 (resp. 6 and 11), respectively. This is represented by $\operatorname{arcs} x_{3,3,2}$ and $x_{5,3,6}$, respectively. Then, arcs $d_{3,11}, d_{3,12}$ and $d_{3,13}$ model an idle time from time 11 until the last available time of berth 3, i.e., time 14 . The same reasoning applies to berths 1 (that handles vessel 1) and 2 (that handles vessels 2 and 4).

\subsection{Equivalence between the mathematical models}

All three formulations, GSPP (1)-(4), TI (5)-(8) and AF (9)-(13), model the DBAP by means of a pseudo-polynomial number of variables. Indeed, in all formulations, the main decision variable indicates the assignment of a vessel to a berth at a given starting time.
It is not surprising thus that the three formulations are all equivalent to one another, i.e., they have the same continuous relaxation value. We skip a formal mathematical proof but give the reader a hint of this equivalence.

Let us first address the relation between GSPP and TI. In the GSPP model, set $\Omega$ contains all feasible assignments of a vessel to a (berth, time) position, and matrices $A^{n \times|\Omega|}$ and $B^{K \times|\Omega|}$, with $K=$ $\sum_{k \in M}\left(e_{k}-s_{k}\right)$, indicate which vessel and (berth, time) positions, respectively, are associated with each variable $x_{\omega}$, for $\omega \in \Omega$. Consider again the example of Table 1 and Fig. 1 . The first possible assignment is that of vessel 1 to berth 1 at time 3 (as both vessel and berth time-windows start in 3). For TI, this is simply represented by variable $x_{113}$. For GSPP, this is instead represented by variable $x_{1}$ and its associated $A$ entries satisfying $A_{11}=1$ and $A_{i 1}=0$ for all $i \neq 1$, and $B$ entries satisfying $B_{p 1}=1$ for $p=1,2, \ldots, 7$ (corresponding to times between 3 and 9 ) and $B_{p 1}=0$ for $p=8,9$ (corresponding to times 10 and 11 ). The only entry taking the value 1 for $A, A_{11}$, indicates in constraint (2) that if $x_{1}$ is chosen, then vessel 1 has been assigned. The same result is obtained for TI by constraints (6) when $x_{113}$ takes the value 1 . The entries taking the value 1 in $B$ indicate in constraints (3) that if $x_{1}$ is chosen then berth 1 is busy until time 10 . The same result is obtained for TI by (7) when $x_{113}$ takes the value 1 . Extending this reasoning to all assignments, one can deduce that (2) can be directly mapped into (6), and (3) into (7). In addition to that, the domains of the variables are identical, as imposed by (4) and (8), so it follows that GSPP and TI are equivalent.

Concerning the relation between $\mathrm{TI}$ and $\mathrm{AF}$, (Valério de Carvalho, 2002) proved that the two formulations are equivalent when applied to the cutting stock problem. Recently, a similar proof has been used by Kramer, Dell'Amico, and Iori (2019) to prove the equivalence of $\mathrm{TI}$ and $\mathrm{AF}$ for the scheduling problem of minimizing total weighted completion time on identical parallel machines. As the proposed TI and AF formulations for the DBAP rely on the same principles of the ones for the cutting stock and the parallel machine scheduling problem, we refer the reader to these works for a proof of equivalence.

It follows that the three formulations are equivalent. Despite this fact, their computational performances are remarkably different. This can be explained by a number of factors. Firstly, for GSPP the computation of the initial matrices $A$ and $B$ can be very memory consuming, and even prohibitive for very large instances. Secondly, it is known that commercial solvers are very sensitive to model details and initial conditions (see, e.g., Fischetti \& Monaci, 2014; Lalla-Ruiz \& Voß, 2016a; Lodi \& Tramontani, 2013). Changes in variables and constraints can thus deeply affect the model performance. Lastly, additional improvement techniques, like those discussed in the next two sections, may render even larger the difference between the performance of the models. All these behaviors can be observed in detail in our computational evaluation in Section 5.

\subsection{Modeling improvements - grouping identical berths and vessels}

The number of incoming vessels arriving at ports every day is increasing (e.g., Brooks \& Faust 2018), some of those vessels may share similar features (i.e., same container workload, timewindows, or priority) as can be checked, for example, in the problem instances based on the port of Gioia Tauro provided by Cordeau et al. (2005). On the other hand, a container terminal can either have some identical (or very similar) berths in terms of productivity and availability windows. Therefore, in this section we introduce new model improvements by considering some problem features like identical berths and vessels. We formalize the necessary conditions that berths and vessels have to comply with in order to be considered identical. Through their proper identifica- 


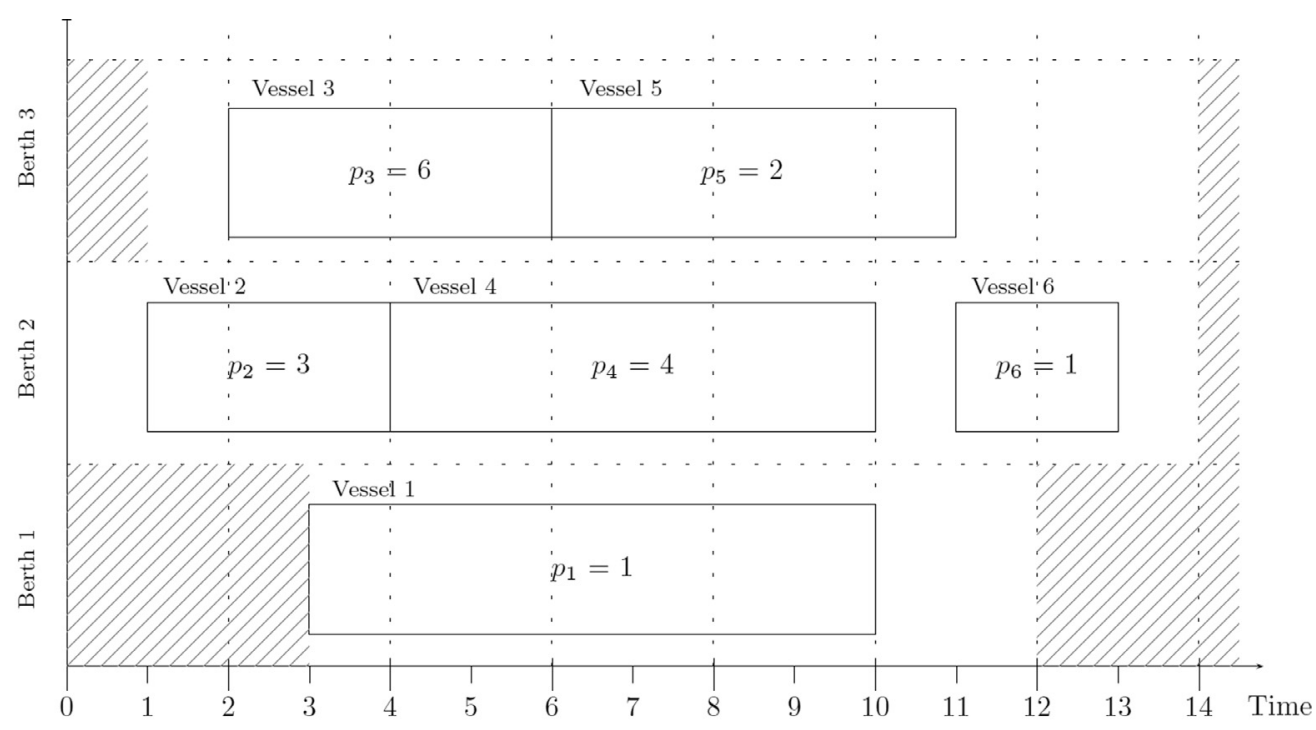

\footnotetext{
\begin{tabular}{l|l} 
Vessel 1: \\
Vessel 2: \\
Vessel 3: \\
in & Vessel 4: \\
of & Vessel 5: \\
& Vessel 6:
\end{tabular}
}

Fig. 1. Example of a DBAP solution with six vessels and three berths. Hatched areas represent berth unavailability due to input time-windows.

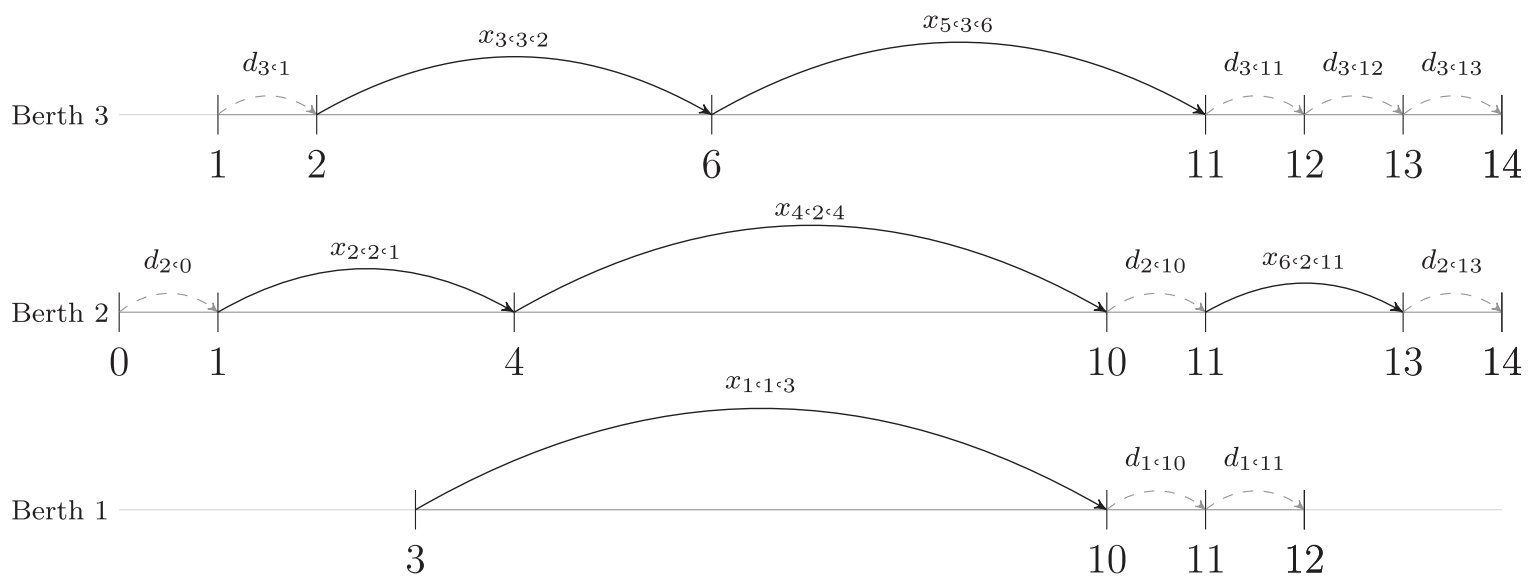

Fig. 2. AF solution for the instance given in Fig. 1 and Table 1.

tion and handling, we aim at reducing the number of variables and constraints of a given model. In the following, we define and indicate how to extract and integrate that information in a preprocessing step before starting to solve the DBAP.

In the DBAP, berths can be compared in terms of their features, i.e., time-windows and processing speed for serving incoming vessels. Thus, subsets of berths operating at the same service speed for the same vessels and sharing the same time-windows can be grouped as identical. This is formally defined by the below definitions.

Definition 1. Two berths $k \in M$ and $l \in M, k \neq l$, are considered identical if the following conditions are satisfied: $s_{k}=s_{l}, e_{k}=e_{l}$, and $\rho_{i k}=\rho_{i l}$ for all $i \in N$.

Definition 2. A berth type is defined by those berth features that allow creating groups of identical berths. The set of berth types is indicated by $M^{\prime}$, such that $M^{\prime} \subseteq M$, and $M^{\prime}=M$ when no identical berths are detected. Additionally, for each berth type $k \in M^{\prime}$, a re- source amount $a_{k}$ is defined as the number of berths of each berth type. Note that $\sum_{k \in M^{\prime}} a_{k}=m$.

Similarly, it is also expected that incoming vessels might have the same features in terms of required service times for the same berth assignment, priorities, and time-windows, and can be consequently considered identical. Formally:

Definition 3. Two vessels $i \in N$ and $j \in N, i \neq j$, are identical if the following conditions are satisfied: $t_{i}=t_{j}, t_{i}^{\prime}=t_{j}^{\prime}, p_{i}=p_{j}$, and $\rho_{i k}=$ $\rho_{j k} \forall k \in M$.

Definition 4. A vessel type is defined by those vessel features that allow to create groups of identical vessels. The set of vessel types is indicated by $N^{\prime}$, such that $N^{\prime} \subseteq N$, and $N^{\prime}=N$ when no identical vessels are detected. The total number of vessels of type $i \in N^{\prime}$ is given by $b_{i}$, with $\sum_{i \in N^{\prime}} b_{i}=n$. 
An example of Definition 4 is as follows. Suppose we have an instance with $N=\{1,2,3,4,5\}$ where vessels 1 and 4 are identical according to Definition 3 , then set $N^{\prime}$ is equal to $\{1,2,3$, 5\} with $b_{1}=2$. Thus, $\sum_{i \in N^{\prime}} b_{i}=5$. This example also applies to Definitions 1 and 2, when treating berths.

Once sets $M^{\prime}$ and $N^{\prime}$ have been defined, one can easily modify the previous formulations to incorporate and make use of this information. Since including this reduction is similar to all formulations, in the following we only show it in the AF formulation (9)(13).

$\left(\mathrm{AF}_{+}\right) \quad \min \sum_{i \in N^{\prime}} \sum_{k \in M^{\prime}} \sum_{t=l_{i k}}^{u_{i k}-\rho_{i k}} p_{i} x_{i k t}\left(t+\rho_{i k}-t_{i}\right)$

subject to

$\sum_{k \in M^{\prime}} \sum_{t=l_{i k}}^{u_{i k}-\rho_{i k}} x_{i k t}=b_{i} \quad i \in N^{\prime}$,

$\sum_{i \in N^{\prime}} x_{i k t}+d_{k t}-\sum_{i \in N^{\prime}} x_{i, k, t-\rho_{i k}}-d_{k, t-1}=\left\{\begin{array}{ll}a_{k}, & \text { if } t=s_{k} \\ -a_{k}, & \text { if } t=e_{k} \\ 0, & \text { otherwise }\end{array} \quad \begin{array}{r}k \in M^{\prime}, \\ t=s_{k}, \ldots, e_{k},\end{array}\right.$

$$
\begin{gathered}
x_{i k t} \in\left\{0, \ldots, \min \left\{a_{k}, b_{i}\right\}\right\} \quad \begin{array}{c}
i \in N^{\prime}, k \in M^{\prime}, \\
t=l_{i k}, \ldots, u_{i k}-\rho_{i k},
\end{array} \\
0 \leq d_{k t} \leq a_{k} \quad \begin{array}{c}
k \in M^{\prime}, \\
t=s_{k}, \ldots, e_{k}-1 .
\end{array}
\end{gathered}
$$

Constraints (15) now take into account that a vessel type $i \in N^{\prime}$ should be served $b_{i}$ times and constraints (16) allow a berth type $k \in M^{\prime}$ to serve at most $a_{k}$ vessels simultaneously. In constraints (17), variables $x_{i k t}$ are now integer and upper bounded by $\min \left\{a_{k}, b_{i}\right\}$ while variables $d_{k t}$ are still continuous, but now upper bounded by $a_{k}$.

\subsection{Modeling improvements - reduced-cost variable-fixing algorithm}

This subsection presents a reduced-cost-based variable-fixing procedure aimed at enhancing the starting conditions of the optimization models. Variable-fixing strategies have been studied in Savelsbergh (1994) and approaches considering them have been widely applied to combinatorial optimization problems.

Our method attempts to reduce the number of variables of a mathematical model by using information given by the optimal solution of the linear model relaxation and by a heuristic DBAP solution. For convenience, we denote an instance of our DBAP problem as $P$, its optimal solution as $x^{*}$, a feasible solution as $x^{U B}$ with objective value of $z\left(x^{U B}\right)$, and the linear relaxation of $P$ as $L P(P)$ with an optimal solution $x^{L P}$ and objective value of $z\left(x^{L P}\right)$. Moreover, related to $x^{L P}$ we denote the reduced costs corresponding to variables $x_{i}^{L P}$ as $\bar{c}_{i}$. Bearing in mind such notation, we state that a variable $x_{i}$ can be fixed to zero in the model if the following condition holds:

$\bar{c}_{i}>z\left(x^{U B}\right)-z\left(x^{L P}\right)-1$.

Suppose indeed there is a non-basic variable $x_{i}$ whose reduced cost $\bar{c}_{i}$ is higher than $z\left(x^{U B}\right)-z\left(x^{L P}\right)-1$. Then, if $x_{i}$ enters the basis with one unit, the current LP objective value $z\left(x^{L P}\right)$ will increase by $\bar{c}_{i}$, thus obtaining $z\left(x^{L P}\right)+\bar{c}_{i}>z\left(x^{U B}\right)-1$. Therefore, any integer solution containing variable $x_{i}$ will have cost at least $\left\lceil z\left(x^{L P}\right)+\bar{c}_{i}\right\rceil \geq z\left(x^{U B}\right)$. We can thus remove $x_{i}$ from the model as we are only interested in solutions that could improve the current incumbent value $z\left(x^{U B}\right)$. This condition is formalized as follows:

Proposition 1. A non-basic variable $x_{i}$ can be removed from the model if its reduced cost $\bar{c}_{i}$ satisfies inequality (19).

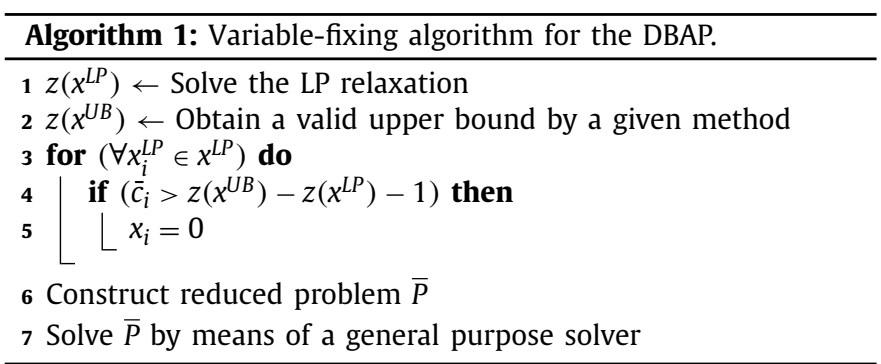

Table 2

Summary of benchmark instances.

\begin{tabular}{llll}
\hline Benchmark set & \#instances & N & M \\
\hline Cordeau et al. (2007) & 30 & $\{60\}$ & $\{13\}$ \\
Lalla-Ruiz et al. (2012) & 90 & $\{30,40,55,60\}$ & $\{3,5,7,10\}$ \\
Nishi et al. (2016) & 50 & $\{80,90,100,120,150\}$ & $\{10,13,15\}$ \\
New proposed & 20 & $\{200,250\}$ & $\{15,20\}$ \\
\hline Total & 190 & & \\
\hline
\end{tabular}

Algorithm 1 describes the overall reduced-cost variable-fixing procedure. At step 1, the linear relaxation of a given DBAP instance is solved. A feasible solution is obtained through a heuristic procedure at step 2 . The variable-fixing is applied at steps $3-5$ by considering inequality (19). After that, a reduced problem $\bar{P}$ is obtained and then solved. This preprocessing procedure requires having tight bounds in order to have a certain impact on the solving performance. Thus, in our current work, we use the state-of-theart heuristic technique by Lalla-Ruiz et al. (2016) to obtain highquality $z\left(x^{U B}\right)$ values.

\section{Computational results}

This section presents the computational experiments carried out for assessing the performance of the proposed formulations. The models were coded in $\mathrm{C}++$ and solved on a computer equipped with an Intel i5 $3.20 \mathrm{GHz}$ and $16 \mathrm{~GB}$ of RAM running under Windows 10 operating system. The models were solved with IBM CPLEX 12.8, using a single thread, and a time limit of 2 hours. The method used for generating the upper bounds is the one provided in Lalla-Ruiz et al. (2016).

\subsection{Benchmark instances}

In this work, we use the problem instances proposed in the literature by Cordeau et al. (2005), Lalla-Ruiz et al. (2012) and Nishi et al. (2016). Among those proposed by Cordeau et al. (2005), we consider the large-sized ones, which contain 60 vessels and 13 berths. Those instances were generated by taking into account a statistical analysis of the traffic and berth allocation data at the maritime container terminal of Gioia Tauro (Italy) and were also studied in Cordeau et al. (2007). Moreover, we tackle the instances proposed by Lalla-Ruiz et al. (2012) that could not be solved to optimality by the computer used to conduct their computational experiments. This set contains 90 instances with up to 60 vessels and 7 berths. We have also used the recently proposed problem instances by Nishi et al. (2016) that consider more congested scenarios with up to 150 vessels and 15 berths. In addition to that, we created 20 new very large instances having up to 250 vessels and 20 berths. All instances are available at http://github.com/elalla/ DBAP/tree/master/Instances_Kramer-Lalla-Ruiz-Iori-Voss/. Table 2 summarizes all the instances used in this work. 
Table 3

Instances with identical vessels and berths.

\begin{tabular}{llllllll}
\hline$n$ & $m$ & $\left|N^{\prime}\right|$ & $\left|M^{\prime}\right|$ & \#inst & \#IV & \#IB & \#IV+IB \\
\hline \multirow{2}{*}{30} & 3 & 24.8 & 3 & 10 & 10 & 0 & 0 \\
& 5 & 24.8 & 4 & 10 & 10 & 10 & 10 \\
40 & 5 & 24.8 & 4 & 10 & 10 & 10 & 10 \\
& 7 & 24.9 & 6 & 10 & 10 & 10 & 10 \\
55 & 5 & 24.8 & 4 & 10 & 10 & 10 & 10 \\
& 7 & 24.9 & 6 & 10 & 10 & 10 & 10 \\
& 10 & 24.9 & 7 & 10 & 10 & 10 & 10 \\
60 & 5 & 24.8 & 4 & 10 & 10 & 10 & 10 \\
& 7 & 35.0 & 6 & 10 & 10 & 10 & 10 \\
& 13 & 59.7 & 5 & 30 & 7 & 30 & 7 \\
80 & 10 & 79.0 & 10 & 10 & 1 & 0 & 0 \\
\hline \multirow{2}{*}{ Total } & & & & 130 & 98 & 110 & 87 \\
\hline
\end{tabular}

\subsection{Computational experiments on the instances from the literature}

In this section, we report and discuss the results obtained by means of the previously introduced formulations (see Section 4). Namely, we compare the performance of GSPP (i.e., (1)-(4)), TI (i.e., (5)-(8)) and AF (i.e., (9)-(13)) formulations. In addition, we assess the contributions of the improvements provided in Sections 4.5 and 4.6. Thus, in the tables, the models incorporating the improvements presented in Section 4.5 are tagged with a "+", while those also considering the reduced-cost-based variable-fixing procedure presented in Section 4.6 are indicated by a " rc ".

In Table 3, we show the sets of instances grouped by $(n, m)$, where there exists at least one instance with identical vessels or berths. Columns $\left|N^{\prime}\right|$ and $\left|M^{\prime}\right|$ indicate, respectively, the average number of vessel and berth types among the instances with identical vessels or berths. Columns \#inst, \#IV, \#IB and \#IV +IB show the total number of instances per group and the number of instances with identical vessels, identical berths and identical vessels and berths, respectively. For example, the group of instances with $(n, m)=(50,5)$ originally contains 55 vessels and 5 berths, but, on average, there are 24.8 and 4 types of vessels and berths, respectively. In particular, all instances from this group have identical vessels and berths. From this table, it is possible to notice that for the 50 instances proposed by Nishi et al. (2016), only one has identical berths. This is explained in part by the instance generation scheme used by Nishi et al. (2016) that considers a different distribution on the handling times and forbidden berths (i.e., a vessel cannot berth at some berths). The presence of heterogeneous sets of instances allows us to evaluate the performance of our algorithms on different scenarios. In particular, the instances with identical berths or vessels make it possible to evaluate the impact of the preprocessing of Section 4.5 (evaluated in Tables 4 and 5).

Table 4 shows a summary of the size of the models in terms of average number of variables (cols) and constraints (rows) reported in thousands. It is worth mentioning that the results shown for the reduced-cost variable-fixing methods represent the size of the reduced mixed integer linear programming formulation obtained after fixing the respective variables to zero. As can be seen in the table, the improvements proposed in this work enable relevant reductions of the model sizes. In this regard, it can be noticed that grouping similar berths and vessels does not always lead to a model size reduction. On the contrary, using the variable-fixing approach results in relevant reductions in all cases. For instance, for the largest instances, the standard models have more than 600 thousand variables on average, while this value can be reduced to nearly 200 thousand in some cases. It is also worth mentioning that AF needs more variables to model the problem than GSPP and TI. This fact is due to the use of the continuous idle variables $d_{i k}$.

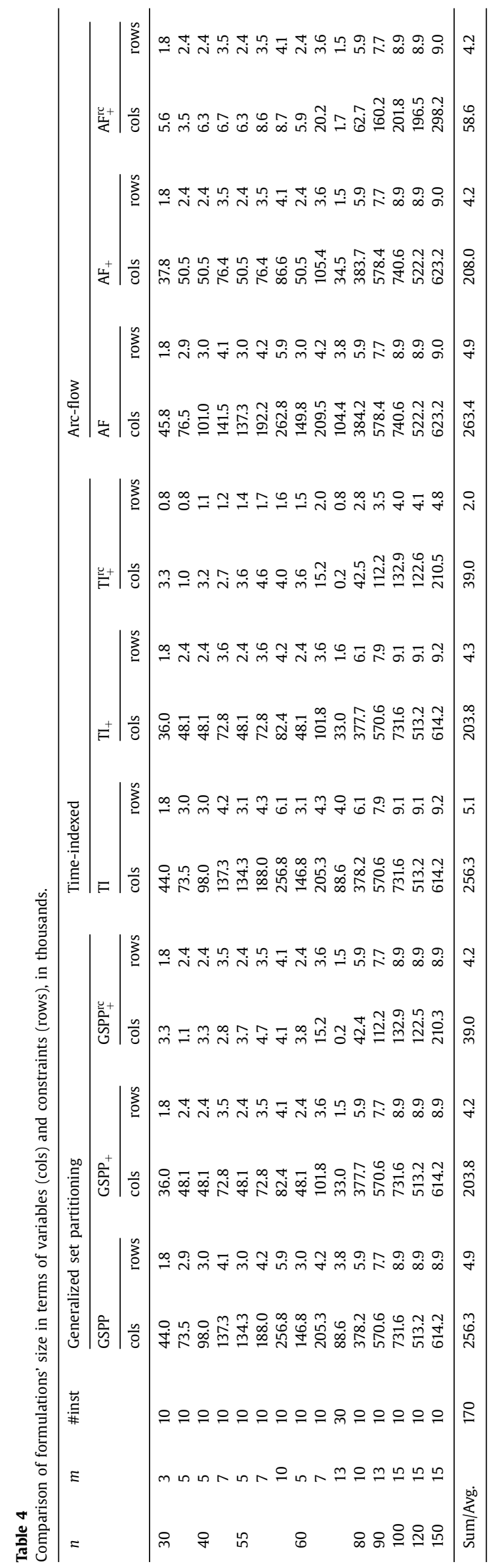


Table 5

Comparison of formulations with and without grouping vessels and berths.

\begin{tabular}{|c|c|c|c|c|c|c|c|c|c|c|c|c|c|c|c|c|c|c|c|c|}
\hline \multirow[t]{3}{*}{$n$} & \multirow[t]{3}{*}{$m$} & \multirow[t]{3}{*}{ \#inst } & \multicolumn{6}{|c|}{ Generalized set partitioning } & \multicolumn{6}{|c|}{ Time-indexed } & \multicolumn{6}{|c|}{ Arc-flow } \\
\hline & & & \multicolumn{2}{|c|}{ GSPP } & \multicolumn{2}{|c|}{$\mathrm{GSPP}_{+}$} & \multicolumn{2}{|c|}{$\operatorname{red}(\%)$} & \multicolumn{2}{|l|}{ TI } & \multicolumn{2}{|l|}{$\mathrm{TI}_{+}$} & \multicolumn{2}{|c|}{$\operatorname{red}(\%)$} & \multicolumn{2}{|l|}{$\mathrm{AF}$} & \multicolumn{2}{|l|}{$\mathrm{AF}_{+}$} & \multicolumn{2}{|c|}{$\operatorname{red}(\%)$} \\
\hline & & & opt & $\mathrm{t}(\mathrm{s})$ & opt & $\mathrm{t}(\mathrm{s})$ & cols & rows & opt & $\mathrm{t}(\mathrm{s})$ & opt & $\mathrm{t}(\mathrm{s})$ & cols & rows & opt & $\mathrm{t}(\mathrm{s})$ & opt & $\mathrm{t}(\mathrm{s})$ & cols & rows \\
\hline \multirow[t]{2}{*}{30} & 3 & 10 & 10 & 12.1 & 10 & 9.8 & 18.2 & 0.2 & 10 & 13.0 & 10 & 9.8 & 18.2 & 0.3 & 10 & 19.0 & 10 & 15.3 & 17.5 & 0.3 \\
\hline & 5 & 10 & 10 & 14.4 & 10 & 10.1 & 34.5 & 19.9 & 10 & 10.7 & 10 & 9.4 & 34.5 & 20.0 & 10 & 4.8 & 10 & 2.9 & 33.9 & 20.0 \\
\hline \multirow[t]{2}{*}{40} & 5 & 10 & 10 & 43.8 & 10 & 16.5 & 50.9 & 20.2 & 10 & 49.8 & 10 & 16.0 & 50.9 & 20.2 & 10 & 63.2 & 10 & 13.7 & 50.0 & 20.2 \\
\hline & 7 & 10 & 10 & 38.2 & 10 & 22.6 & 47.0 & 14.5 & 10 & 26.4 & 10 & 14.1 & 47.0 & 14.5 & 10 & 9.2 & 10 & 4.7 & 46.0 & 14.5 \\
\hline \multirow[t]{3}{*}{55} & 5 & 10 & 10 & 64.8 & 10 & 15.3 & 64.2 & 20.6 & 10 & 69.1 & 10 & 12.5 & 64.2 & 20.6 & 10 & 25.1 & 10 & 11.0 & 63.2 & 20.6 \\
\hline & 7 & 10 & 10 & 103.8 & 10 & 29.1 & 61.3 & 14.8 & 10 & 106.9 & 10 & 18.1 & 61.3 & 14.8 & 10 & 56.5 & 10 & 14.0 & 60.3 & 14.8 \\
\hline & 10 & 10 & 10 & 110.6 & 10 & 22.4 & 67.9 & 30.2 & 10 & 48.5 & 10 & 10.0 & 67.9 & 30.2 & 10 & 31.2 & 10 & 6.2 & 67.0 & 30.2 \\
\hline \multirow[t]{3}{*}{60} & 5 & 10 & 10 & 100.0 & 10 & 21.6 & 67.2 & 20.8 & 10 & 81.4 & 10 & 17.0 & 67.2 & 20.8 & 10 & 68.2 & 10 & 16.0 & 66.3 & 20.8 \\
\hline & 7 & 10 & 10 & 220.7 & 10 & 71.1 & 50.4 & 14.7 & 10 & 240.5 & 10 & 91.7 & 50.4 & 14.7 & 10 & 175.0 & 10 & 79.4 & 49.7 & 14.7 \\
\hline & 13 & 30 & 30 & 12.5 & 30 & 1.9 & 62.8 & 60.6 & 30 & 3.3 & 30 & 0.9 & 62.8 & 60.6 & 30 & 4.1 & 30 & 0.9 & 66.9 & 60.6 \\
\hline 80 & 10 & 10 & 10 & 157.6 & 10 & 165.4 & 0.1 & 0.0 & 10 & 113.6 & 10 & 135.8 & 0.1 & 0.0 & 10 & 141.6 & 10 & 110.1 & 0.1 & 0.0 \\
\hline \multicolumn{2}{|c|}{ Sum/Avg. } & 130 & 130 & 69.5 & 130 & 30.0 & 46.3 & 26.2 & 130 & 59.2 & 130 & 25.9 & 46.3 & 26.4 & 130 & 46.6 & 130 & 21.3 & 46.8 & 26.2 \\
\hline
\end{tabular}

Table 6

Comparison of formulations with and without variable-fixing.

\begin{tabular}{|c|c|c|c|c|c|c|c|c|c|c|c|c|c|c|c|c|c|}
\hline \multirow[t]{3}{*}{$n$} & \multirow[t]{3}{*}{$m$} & \multirow[t]{3}{*}{ \#inst } & \multicolumn{5}{|c|}{ Generalized set partitioning } & \multicolumn{5}{|c|}{ Time-indexed } & \multicolumn{5}{|c|}{ Arc-flow } \\
\hline & & & \multicolumn{2}{|c|}{$\mathrm{GSPP}_{+}$} & \multicolumn{2}{|c|}{$\mathrm{GSPP}_{+}^{\mathrm{rc}}$} & \multirow{2}{*}{$\begin{array}{l}\text { red(\%) } \\
\text { cols }\end{array}$} & \multicolumn{2}{|l|}{$\mathrm{TI}_{+}$} & \multicolumn{2}{|l|}{$\mathrm{TI}_{+}^{\mathrm{rc}}$} & \multirow{2}{*}{$\begin{array}{l}\operatorname{red}(\%) \\
\text { cols }\end{array}$} & \multicolumn{2}{|l|}{$\mathrm{AF}_{+}$} & \multicolumn{2}{|l|}{$\mathrm{AF}_{+}^{\mathrm{rc}}$} & \multirow{2}{*}{$\begin{array}{l}\operatorname{red}(\% \\
\text { cols }\end{array}$} \\
\hline & & & opt & $\mathrm{t}(\mathrm{s})$ & opt & $\mathrm{t}(\mathrm{s})$ & & opt & $\mathrm{t}(\mathrm{s})$ & opt & $\mathrm{t}(\mathrm{s})$ & & opt & $\mathrm{t}(\mathrm{s})$ & opt & $\mathrm{t}(\mathrm{s})$ & \\
\hline \multirow[t]{2}{*}{30} & 3 & 10 & 10 & 9.8 & 10 & 7.2 & 90.9 & 10 & 9.8 & 10 & 2.7 & 90.9 & 10 & 15.3 & 10 & 4.2 & 85.3 \\
\hline & 5 & 10 & 10 & 10.1 & 10 & 10.4 & 97.7 & 10 & 9.4 & 10 & 1.3 & 97.8 & 10 & 2.9 & 10 & 0.9 & 93.1 \\
\hline \multirow[t]{2}{*}{40} & 5 & 10 & 10 & 16.5 & 10 & 10.9 & 93.1 & 10 & 16.0 & 10 & 3.4 & 93.3 & 10 & 13.7 & 10 & 1.7 & 87.6 \\
\hline & 7 & 10 & 10 & 22.6 & 10 & 20.9 & 96.2 & 10 & 14.1 & 10 & 2.1 & 96.2 & 10 & 4.7 & 10 & 1.7 & 91.2 \\
\hline \multirow[t]{3}{*}{55} & 5 & 10 & 10 & 15.3 & 10 & 11.5 & 92.3 & 10 & 12.5 & 10 & 4.2 & 92.5 & 10 & 11.0 & 10 & 1.8 & 87.5 \\
\hline & 7 & 10 & 10 & 29.1 & 10 & 23.8 & 93.5 & 10 & 18.1 & 10 & 5.8 & 93.7 & 10 & 14.0 & 10 & 3.6 & 88.8 \\
\hline & 10 & 10 & 10 & 22.4 & 10 & 26.9 & 95.0 & 10 & 10.0 & 10 & 3.3 & 95.1 & 10 & 6.2 & 10 & 2.6 & 89.9 \\
\hline \multirow[t]{3}{*}{60} & 5 & 10 & 10 & 21.6 & 10 & 15.8 & 92.2 & 10 & 17.0 & 10 & 6.7 & 92.6 & 10 & 16.0 & 10 & 2.3 & 88.3 \\
\hline & 7 & 10 & 10 & 71.1 & 10 & 71.3 & 85.1 & 10 & 91.7 & 10 & 45.8 & 85.1 & 10 & 79.4 & 10 & 26.3 & 80.8 \\
\hline & 13 & 30 & 30 & 1.9 & 30 & 3.9 & 99.4 & 30 & 0.9 & 30 & 1.7 & 99.4 & 30 & 0.9 & 30 & 1.9 & 95.0 \\
\hline 80 & 10 & 10 & 10 & 165.4 & 10 & 199.0 & 88.8 & 10 & 135.8 & 10 & 32.9 & 88.7 & 10 & 110.1 & 10 & 24.2 & 83.7 \\
\hline 90 & 13 & 10 & 10 & 643.7 & 10 & 930.9 & 80.3 & 10 & 327.8 & 10 & 203.6 & 80.3 & 10 & 488.8 & 10 & 218.5 & 72.3 \\
\hline 100 & 15 & 10 & 10 & 1194.0 & 10 & 1776.8 & 81.8 & 10 & 797.2 & 10 & 546.1 & 81.8 & 10 & 856.1 & 10 & 478.9 & 72.7 \\
\hline 120 & 15 & 10 & 9 & 1791.7 & 10 & 1756.4 & 76.1 & 10 & 1063.8 & 10 & 1418.2 & 76.1 & 10 & 1231.2 & 10 & 930.8 & 62.4 \\
\hline 150 & 15 & 10 & 5 & 5637.7 & 5 & 5545.1 & 65.8 & 3 & 6067.5 & 3 & 5902.5 & 65.7 & 4 & 5646.1 & 6 & 5570.7 & 52.5 \\
\hline \multicolumn{2}{|c|}{ Sum/Avg. } & 170 & 164 & 568.0 & 165 & 612.9 & 80.9 & 163 & 505.5 & 163 & 481.4 & 80.9 & 164 & 499.9 & 166 & 427.9 & 71.9 \\
\hline
\end{tabular}


Table 7

Results for large-size instances considering 120 vessels. In boldface, best upper bound value for each instance.

\begin{tabular}{|c|c|c|c|c|c|c|c|c|c|c|c|c|c|c|c|c|c|c|c|c|c|c|c|c|c|c|}
\hline \multirow[t]{3}{*}{$n$} & \multirow[t]{3}{*}{$m$} & \multirow[t]{3}{*}{ id } & \multicolumn{8}{|c|}{ Generalized set partitioning } & \multicolumn{8}{|c|}{ Time-indexed } & \multicolumn{8}{|c|}{ Arc-flow } \\
\hline & & & \multicolumn{4}{|l|}{$\mathrm{GSPP}_{+}$} & \multicolumn{4}{|l|}{$\mathrm{GSPP}_{+}^{r c}$} & \multicolumn{4}{|l|}{$\mathrm{TI}_{+}$} & \multicolumn{4}{|l|}{$\mathrm{Tl}_{+}^{r c}$} & \multicolumn{4}{|l|}{$\mathrm{AF}_{+}$} & \multicolumn{4}{|l|}{$\mathrm{AF}_{+}^{r c}$} \\
\hline & & & $\mathrm{lb}$ & ub & gap(\%) & $t(s)$ & $\mathrm{lb}$ & ub & gap $(\%)$ & $\mathrm{t}(\mathrm{s})$ & $\mathrm{lb}$ & ub & gap(\%) & $\mathrm{t}(\mathrm{s})$ & $\mathrm{lb}$ & ub & gap(\%) & $\mathrm{t}(\mathrm{s})$ & $\mathrm{lb}$ & ub & gap $(\%)$ & $\mathrm{t}(\mathrm{s})$ & $\mathrm{lb}$ & ub & gap(\%) & $t(s)$ \\
\hline \multirow[t]{10}{*}{120} & 15 & 01 & 4065.0 & 4065 & 0.00 & 924.9 & 4065.0 & 4065 & 0.00 & 1145.5 & 4065.0 & 4065 & 0.00 & 1185.4 & 4065.0 & 4065 & 0.00 & 283.3 & 4065.0 & 4065 & 0.00 & 756.5 & 4065.0 & 4065 & 0.00 & 152.4 \\
\hline & & 02 & 3653.0 & 3653 & 0.00 & 550.5 & 3653.0 & 3653 & 0.00 & 858.6 & 3653.0 & 3653 & 0.00 & 123.5 & 3653.0 & 3653 & 0.00 & 81.9 & 3653.0 & 3653 & 0.00 & 47.6 & 3653.0 & 3653 & 0.00 & 29.9 \\
\hline & & 03 & 3756.0 & 3756 & 0.00 & 774.0 & 3756.0 & 3756 & 0.00 & 1007.6 & 3756.0 & 3756 & 0.00 & 527.7 & 3756.0 & 3756 & 0.00 & 1139.9 & 3756.0 & 3756 & 0.00 & 98.2 & 3756.0 & 3756 & 0.00 & 255.1 \\
\hline & & 04 & 3211.0 & 3211 & 0.00 & 1390.5 & 3211.0 & 3211 & 0.00 & 1711.8 & 3211.0 & 3211 & 0.00 & 1652.4 & 3211.0 & 3211 & 0.00 & 1433.1 & 3211.0 & 3211 & 0.00 & 1003.6 & 3211.0 & 3211 & 0.00 & 2336.2 \\
\hline & & 05 & 4294.5 & 4298 & 0.08 & 7200.0 & 4296.0 & 4296 & 0.00 & 4208.2 & 4296.0 & 4296 & 0.00 & 2303.1 & 4296.0 & 4296 & 0.00 & 4721.6 & 4296.0 & 4296 & 0.00 & 3727.2 & 4296.0 & 4296 & 0.00 & 3032.6 \\
\hline & & 06 & 4512.0 & 4512 & 0.00 & 758.2 & 4512.0 & 4512 & 0.00 & 1977.0 & 4512.0 & 4512 & 0.00 & 1853.1 & 4512.0 & 4512 & 0.00 & 2173.5 & 4512.0 & 4512 & 0.00 & 1680.9 & 4512.0 & 4512 & 0.00 & 305.4 \\
\hline & & 07 & 3463.0 & 3463 & 0.00 & 596.0 & 3463.0 & 3463 & 0.00 & 779.6 & 3463.0 & 3463 & 0.00 & 126.2 & 3463.0 & 3463 & 0.00 & 108.1 & 3463.0 & 3463 & 0.00 & 36.7 & 3463.0 & 3463 & 0.00 & 61.1 \\
\hline & & 08 & 3872.0 & 3872 & 0.00 & 1202.8 & 3872.0 & 3872 & 0.00 & 1074.1 & 3872.0 & 3872 & 0.00 & 916.4 & 3872.0 & 3872 & 0.00 & 274.2 & 3872.0 & 3872 & 0.00 & 1437.2 & 3872.0 & 3872 & 0.00 & 149.3 \\
\hline & & 09 & 4176.0 & 4176 & 0.00 & 1582.2 & 4176.0 & 4176 & 0.00 & 2054.9 & 4176.0 & 4176 & 0.00 & 1072.5 & 4176.0 & 4176 & 0.00 & 1114.6 & 4176.0 & 4176 & 0.00 & 1974.5 & 4176.0 & 4176 & 0.00 & 1110.1 \\
\hline & & 10 & 3880.0 & 3880 & 0.00 & 2938.3 & 3880.0 & 3880 & 0.00 & 2746.8 & 3880.0 & 3880 & 0.00 & 877.8 & 3880.0 & 3880 & 0.00 & 2851.7 & 3880.0 & 3880 & 0.00 & 1550.1 & 3880.0 & 3880 & 0.00 & 1876.3 \\
\hline Avg. & & & 3888.3 & 3888.6 & 0.01 & 1791.7 & 3888.4 & 3888.4 & 0.00 & 1756.4 & 3888.4 & 3888.4 & 0.00 & 1063.8 & 3888.4 & 3888.4 & 0.00 & 1418.2 & 3888.4 & 3888.4 & 0.00 & 1231.2 & 3888.4 & 3888.4 & 0.00 & 930.8 \\
\hline
\end{tabular}

Table 8

Results for large-size instances considering 150 vessels. In boldface, best upper bound value for each instance.

\begin{tabular}{|c|c|c|c|c|c|c|c|c|c|c|c|c|c|c|c|c|c|c|c|c|c|c|c|c|c|c|}
\hline \multirow[t]{3}{*}{$n$} & \multirow[t]{3}{*}{$m$} & \multirow[t]{3}{*}{ id } & \multicolumn{8}{|c|}{ Generalized set partitioning } & \multicolumn{8}{|c|}{ Time-indexed } & \multicolumn{8}{|c|}{ Arc-flow } \\
\hline & & & \multicolumn{4}{|l|}{$\mathrm{GSPP}_{+}$} & \multicolumn{4}{|l|}{$\mathrm{GSPP}_{+}^{\mathrm{rc}}$} & \multicolumn{4}{|l|}{$\mathrm{TI}_{+}$} & \multicolumn{4}{|l|}{$\mathrm{Tl}_{+}^{\mathrm{rc}}$} & \multicolumn{4}{|l|}{$\mathrm{AF}_{+}$} & \multicolumn{4}{|l|}{$\mathrm{AF}_{+}^{\mathrm{rc}}$} \\
\hline & & & $\mathrm{lb}$ & $\mathrm{ub}$ & gap(\%) & $t(s)$ & $\mathrm{lb}$ & ub & gap(\%) & $\mathrm{t}(\mathrm{s})$ & $\mathrm{lb}$ & $\mathrm{ub}$ & gap(\%) & $\mathrm{t}(\mathrm{s})$ & $\mathrm{lb}$ & ub & gap $\%$ ) & $\mathrm{t}(\mathrm{s})$ & $\mathrm{lb}$ & ub & gap(\%) & $t(s)$ & $\mathrm{lb}$ & ub & gap $(\%)$ & $\mathrm{t}(\mathrm{s})$ \\
\hline \multirow[t]{10}{*}{150} & 15 & 01 & 8214.5 & 8225 & 0.13 & tlim & 8214.6 & 8220 & 0.07 & tlim & 8216.2 & 8220 & 0.05 & $\operatorname{tlim}$ & 8214.5 & 8221 & 0.08 & $\operatorname{tlim}$ & 8216.9 & 8220 & 0.04 & $\operatorname{tlim}$ & 8219.0 & 8219 & 0.00 & 7028.1 \\
\hline & & 02 & 6736.8 & 6752 & 0.22 & tlim & 6737.2 & 6748 & 0.16 & tlim & 6736.9 & 6744 & 0.11 & tlim & 6736.6 & 6745 & 0.13 & tlim & 6737.4 & 6746 & 0.13 & tlim & 6737.7 & 6742 & 0.06 & tlim \\
\hline & & 03 & 4655.0 & 4655 & 0.00 & 922.4 & 4655.0 & 4655 & 0.00 & 1175.9 & 4655.0 & 4655 & 0.00 & 284.5 & 4655.0 & 4655 & 0.00 & 212.6 & 4655.0 & 4655 & 0.00 & 51.0 & 4655.0 & 4655 & 0.00 & 66.7 \\
\hline & & 04 & 7303.0 & 7303 & 0.00 & tlim & 7303.0 & 7303 & 0.00 & 5196.4 & 7301.2 & 7305 & 0.05 & $\operatorname{tlim}$ & 7301.1 & 7341 & 0.54 & tlim & 7301.5 & 7309 & 0.10 & $\operatorname{tlim}$ & 7301.5 & 7305 & 0.05 & tlim \\
\hline & & 05 & 6563.0 & 6563 & 0.00 & 2665.3 & 6561.6 & 6563 & 0.02 & tlim & 6561.6 & 6563 & 0.02 & tlim & 6561.5 & 6563 & 0.02 & tlim & 6563.0 & 6563 & 0.00 & 3761.3 & 6563.0 & 6563 & 0.00 & 2258.2 \\
\hline & & 06 & 6347.6 & 6359 & 0.18 & tlim & 6347.5 & 6360 & 0.20 & tlim & 6347.3 & 6375 & 0.43 & tlim & 6347.4 & 6361 & 0.21 & tlim & 6347.6 & 6363 & 0.24 & tlim & 6348.0 & 6360 & 0.19 & $\operatorname{tlim}$ \\
\hline & & 07 & 6343.0 & 6343 & 0.00 & 5503.4 & 6343.0 & 6343 & 0.00 & 5457.1 & 6343.0 & 6343 & 0.00 & 5174.9 & 6343.0 & 6343 & 0.00 & 5713.6 & 6343.0 & 6343 & 0.00 & 4914.7 & 6343.0 & 6343 & 0.00 & 7006.9 \\
\hline & & 08 & 7939.0 & 7940 & 0.01 & tlim & 7940.0 & 7940 & 0.00 & 5364.8 & 7939.0 & 7940 & 0.01 & tlim & 7939.0 & 7940 & 0.01 & tlim & 7939.0 & 7940 & 0.01 & tlim & 7940.0 & 7940 & 0.00 & 7035.3 \\
\hline & & 09 & 8242.0 & 8242 & 0.00 & 4086.0 & 8242.0 & 8242 & 0.00 & 2137.9 & 8242.0 & 8242 & 0.00 & 4815.8 & 8242.0 & 8242 & 0.00 & 2531.1 & 8242.0 & 8242 & 0.00 & 4533.8 & 8242.0 & 8242 & 0.00 & 3414.1 \\
\hline & & 10 & 6012.0 & 6024 & 0.20 & tlim & 6012.9 & 6016 & 0.05 & tlim & 6012.1 & 6016 & 0.07 & tlim & 6012.0 & 6020 & 0.13 & tlim & 6012.4 & 6020 & 0.13 & tlim & 6012.5 & 6016 & 0.06 & $\operatorname{tlim}$ \\
\hline Avg. & & & 6835.6 & 6840.6 & 0.07 & 5637.7 & 6835.7 & 6839.0 & 0.05 & 5533.2 & 6835.4 & 6840.3 & 0.07 & 6067.5 & 6835.2 & 6843.1 & 0.11 & 5885.7 & 6835.8 & 6840.1 & 0.06 & 5646.1 & 6836.2 & 6838.5 & 0.04 & 5560.9 \\
\hline
\end{tabular}




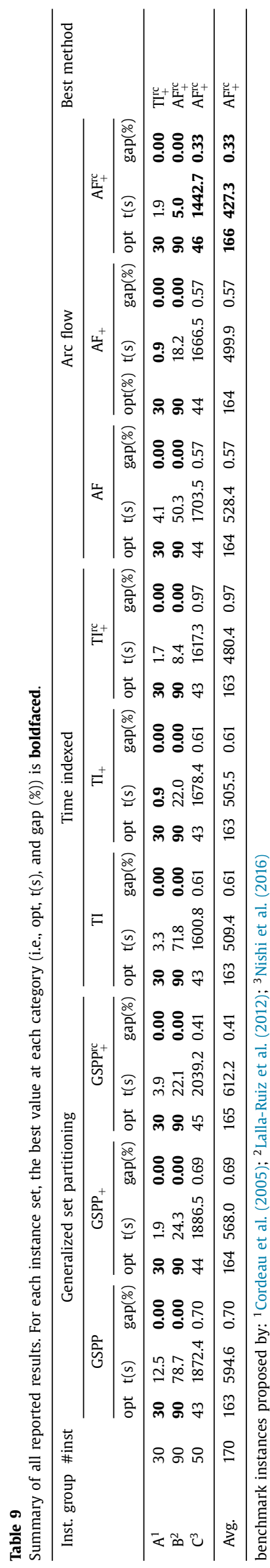

Tables 5 and 6 depict and compare in more detail the contribution achieved by grouping identical vessels and berths as well as by fixing variables, respectively. In these tables, columns opt and $t(s)$ report, per group of instances, the number of problem instances solved to proven optimality and the average execution time in seconds, respectively. In addition, columns cols and rows (only for Table 5) under red(\%) detail the reduction achieved by applying such improvements. The values in boldface indicate the best average execution times for each group of instances where all methods managed to solve all instances to proven optimality.

In Table 5, we report the results for those instances where identical vessels and berths were identified, i.e., instances with up to 80 vessels. Concerning the performance of the studied methods, all of them are able to solve to optimality all 130 instances with up to 80 vessels within the time limit of 2 hours. The results also indicate that grouping identical vessels and berths leads to a significant reduction of more than $60 \%$ in the number of rows and columns. Further, in most cases, the improved models require fewer nodes to be explored, which entails a computational effort reduction. It is relevant to mention that, on average, the computational times of the models without improvements are halved when the improvements are incorporated. On the other hand, the new formulations, TI and AF, exhibit a slightly better performance than GSPP in terms of $t(s)$.

After reporting the benefits of reducing the models by grouping identical vessels and berths, in Table 6 we compare the performance of the reduced-cost variable-fixing procedure on the above studied enhanced models for all problem instances. It is worth mentioning that for the reduced-cost variable-fixing methods column $t(s)$ refers to the execution times for the whole procedure, including the metaheuristic execution times. For detailed results by the metaheuristic, execution times and upper bounds, we refer the reader to Appendix A. Table 6 shows that by applying the reduced-cost variable-fixing technique we can avoid creating, on average, more than $70 \%$ of the initial variables, thus we are able to substantially reduce the formulations' sizes. Despite this huge reduction in the number of variables, the reduction in the execution times is more moderate. Taking the AF results as an example, it can be seen that a variable reduction of $70 \%$ has been achieved while the execution times have been reduced by $14.4 \%$ on average. This can be explained by the fact that there is a time overhead for running the heuristic, solving the linear relaxation and identifying and fixing the variables as well as the fact that the remaining subsequent mathematical model is still difficult to solve.

It is also shown in Table 6 that for instances with 120 and 150 vessels the application of the reduced-cost-based variable-fixing method allows solving more problem instances to optimality. In this regard, this table indicates that the $\mathrm{AF}$ with fixed variables performs better than the other formulations being able to find optimal solutions for 166 out of 170 instances within less computational time. These results are detailed in Tables 7 and 8.

Tables 7 and 8 detail the results obtained for the large-size instances considering 120 and 150 vessels, respectively. For each mathematical formulation and instance, we report the final lower and upper bounds, $l b$ and $u b$, respectively, the percentage gap $g a p(\%)$, and the computational time $t(s)$. An entry tlim indicates that the time limit of two hours is reached. Note that due to all input numbers being integer, in these tables $l b$ could be replaced by $\lceil l b\rceil$, but we opted to keep $l b$ to better highlight the differences among the models.

From Table 7, it can be observed that the use of the variablefixing approach with the GSPP model allows the solver to accelerate and find all the optimal solutions in comparison to the case where this technique is not applied (i.e., instance 120x15-05). Concerning the resolution times, by analyzing instance by instance we can observe that the time required by the variable-fixing method 
Table 10

Comparison of formulations' size in terms of variables (cols) and constraints (rows), in thousands.

\begin{tabular}{|c|c|c|c|c|c|c|c|c|c|c|}
\hline \multirow[t]{3}{*}{$n$} & \multirow[t]{3}{*}{$m$} & \multirow[t]{3}{*}{ \#inst } & \multicolumn{4}{|c|}{ Time-indexed } & \multicolumn{4}{|c|}{ Arc-flow } \\
\hline & & & \multicolumn{2}{|l|}{$\mathrm{TI}_{+}$} & \multicolumn{2}{|l|}{$\mathrm{TI}_{+}^{\mathrm{rc}}$} & \multicolumn{2}{|l|}{$\mathrm{AF}_{+}$} & \multicolumn{2}{|l|}{$\mathrm{AF}_{+}^{\mathrm{rc}}$} \\
\hline & & & cols & rows & cols & rows & cols & rows & cols & rows \\
\hline 200 & 15 & 10 & 1273.7 & 9.2 & 753.5 & 7.6 & 1277.0 & 8.9 & 896.6 & 8.9 \\
\hline 250 & 20 & 10 & 2421.2 & 12.3 & 1341.8 & 9.5 & 2421.3 & 11.8 & 1659.6 & 11.8 \\
\hline \multicolumn{2}{|c|}{ Sum/Avg. } & 20 & 1847.5 & 10.7 & 1047.7 & 8.5 & 1849.1 & 10.3 & 1278.1 & 10.3 \\
\hline
\end{tabular}

Table 11

Results for new large-size instances considering 200 vessels. In boldface, best upper bound value for each instance

\begin{tabular}{|c|c|c|c|c|c|c|c|c|c|c|c|c|c|c|c|c|c|c|}
\hline \multirow[t]{3}{*}{$n$} & \multirow[t]{3}{*}{$m$} & \multirow[t]{3}{*}{ id } & \multicolumn{8}{|c|}{ Time-indexed } & \multicolumn{8}{|l|}{ Arc-flow } \\
\hline & & & \multicolumn{4}{|l|}{$\mathrm{TI}_{+}$} & \multicolumn{4}{|l|}{$\mathrm{TI}_{+}^{\mathrm{rc}}$} & \multicolumn{4}{|l|}{$\mathrm{AF}_{+}$} & \multicolumn{4}{|l|}{$\mathrm{AF}_{+}^{\mathrm{rc}}$} \\
\hline & & & $\mathrm{lb}$ & $\mathrm{ub}$ & gap(\%) & $t(s)$ & $\mathrm{lb}$ & $\mathrm{ub}$ & gap(\%) & $\mathrm{t}(\mathrm{s})$ & $\mathrm{lb}$ & $\mathrm{ub}$ & gap(\%) & $\mathrm{t}(\mathrm{s})$ & $\mathrm{lb}$ & ub & gap(\%) & $\mathrm{t}(\mathrm{s})$ \\
\hline \multirow[t]{10}{*}{200} & \multirow[t]{10}{*}{15} & 01 & 12603.3 & 12613 & $0.08 \%$ & tlim & 12603.3 & 12609 & $0.05 \%$ & tlim & 12603.3 & 12709 & $0.83 \%$ & tlim & 12603.3 & 12709 & $0.83 \%$ & tlim \\
\hline & & 02 & 10319.0 & 10319 & $0.00 \%$ & 6301.8 & 10317.6 & 10319 & $0.01 \%$ & tlim & 10319.0 & 10319 & $0.00 \%$ & 3620.8 & 10319.0 & 10319 & $0.00 \%$ & 1971.0 \\
\hline & & 03 & 11289.8 & 11558 & $2.32 \%$ & 7200.0 & 11295.4 & 11355 & $0.52 \%$ & tlim & 11292.0 & 11558 & $2.30 \%$ & tlim & 11294.4 & 11416 & $1.07 \%$ & tlim \\
\hline & & 04 & 15433.9 & 15480 & $0.30 \%$ & tlim & 15437.4 & 15441 & $0.02 \%$ & tlim & 15441.0 & 15441 & $0.00 \%$ & 6880.6 & 15441.0 & 15441 & $0.00 \%$ & 3671.1 \\
\hline & & 05 & 18164.7 & 18352 & $1.02 \%$ & tlim & 18165.0 & 18352 & $1.02 \%$ & tlim & 18159.4 & 18352 & $1.05 \%$ & tlim & 18165.6 & 18352 & $1.02 \%$ & tlim \\
\hline & & 06 & 16869.0 & 16869 & $0.00 \%$ & 6836.4 & 16869.0 & 16869 & $0.00 \%$ & 6491.5 & 16869.0 & 16869 & $0.00 \%$ & 1015.5 & 16869.0 & 16869 & $0.00 \%$ & 1612.8 \\
\hline & & 07 & 13023.7 & 13226 & $1.53 \%$ & tlim & 13023.7 & 13226 & $1.53 \%$ & tlim & 13023.5 & 13226 & $1.53 \%$ & tlim & 13025.0 & 13226 & $1.52 \%$ & tlim \\
\hline & & 08 & 14176.6 & 14537 & $2.48 \%$ & tlim & 14181.2 & 14537 & $2.45 \%$ & tlim & 14180.4 & 14298 & $0.82 \%$ & tlim & 14180.5 & 14259 & $0.55 \%$ & tlim \\
\hline & & 09 & 18115.8 & 18198 & $0.45 \%$ & tlim & 18118.0 & 18118 & $0.00 \%$ & 1946.9 & 18118.0 & 18118 & $0.00 \%$ & 3879.7 & 18118.0 & 18118 & $0.00 \%$ & 4987.1 \\
\hline & & 10 & 17095.4 & 17263 & $0.97 \%$ & tlim & 17100.9 & 17263 & $0.94 \%$ & tlim & 17100.8 & 17118 & $0.10 \%$ & tlim & 17101.6 & 17134 & $0.19 \%$ & tlim \\
\hline Avg. & & & 14709.1 & 14841.5 & $0.91 \%$ & 7073.8 & 14711.2 & 14808.9 & $0.65 \%$ & 6603.8 & 14710.6 & 14800.8 & $0.66 \%$ & 5859.7 & 14711.7 & 14784.3 & $0.52 \%$ & 5544.2 \\
\hline
\end{tabular}

Table 12

Results for new large-size instances considering 250 vessels. In boldface, best upper bound value for each instance

\begin{tabular}{|c|c|c|c|c|c|c|c|c|c|c|c|c|c|c|c|c|c|c|}
\hline \multirow[t]{3}{*}{$n$} & \multirow[t]{3}{*}{$m$} & \multirow[t]{3}{*}{ id } & \multicolumn{8}{|c|}{ Time-indexed } & \multicolumn{8}{|l|}{ Arc-flow } \\
\hline & & & \multicolumn{4}{|l|}{$\mathrm{TI}_{+}$} & \multicolumn{4}{|l|}{$\mathrm{TI}_{+}^{\mathrm{rc}}$} & \multicolumn{4}{|l|}{$\mathrm{AF}_{+}$} & \multicolumn{4}{|l|}{$\mathrm{AF}_{+}^{\mathrm{rc}}$} \\
\hline & & & $\mathrm{lb}$ & ub & gap(\%) & $\mathrm{t}(\mathrm{s})$ & $\mathrm{lb}$ & ub & gap(\%) & $\mathrm{t}(\mathrm{s})$ & $\mathrm{lb}$ & ub & gap $(\%)$ & $\mathrm{t}(\mathrm{s})$ & $\mathrm{lb}$ & $\mathrm{ub}$ & gap $(\%)$ & $\mathrm{t}(\mathrm{s})$ \\
\hline \multirow[t]{10}{*}{250} & \multirow[t]{10}{*}{20} & 01 & 15632.2 & 15769 & 0.87 & tlim & 15632.4 & 15769 & 0.87 & tlim & 15632.3 & 15769 & 0.87 & tlim & 15632.3 & 15769 & 0.87 & tlim \\
\hline & & 02 & 15774.9 & 15915 & 0.88 & tlim & 15775.1 & 15915 & 0.88 & tlim & 15774.9 & 15915 & 0.88 & tlim & 15775.0 & 15915 & 0.88 & tlim \\
\hline & & 03 & 16518.9 & 16606 & 0.52 & tlim & 16518.8 & 16631 & 0.67 & tlim & 16518.9 & 16724 & 1.23 & tlim & 16518.9 & 16724 & 1.23 & tlim \\
\hline & & 04 & 16422.6 & 16490 & 0.41 & tlim & 16422.6 & 16481 & 0.35 & tlim & 16422.6 & 16509 & 0.52 & tlim & 16422.6 & 16509 & 0.52 & tlim \\
\hline & & 05 & 15661.0 & 15837 & 1.11 & tlim & 15661.0 & 15837 & 1.11 & tlim & 15661.0 & 15837 & 1.11 & tlim & 15661.0 & 15837 & 1.11 & tlim \\
\hline & & 06 & 20060.0 & 20193 & 0.66 & tlim & 20060.0 & 20193 & 0.66 & tlim & 20060.0 & 20060 & 0.00 & 5180.6 & 20060.0 & 20193 & 0.66 & tlim \\
\hline & & 07 & 14283.3 & 14514 & 1.59 & tlim & 14283.3 & 14362 & 0.55 & tlim & 14283.3 & 14514 & 1.59 & tlim & 14283.3 & 14514 & 1.59 & tlim \\
\hline & & 08 & 16303.7 & 16386 & 0.50 & tlim & 16304.1 & 16383 & 0.48 & tlim & 16303.7 & 16498 & 1.18 & tlim & 16303.7 & 16498 & 1.18 & tlim \\
\hline & & 09 & 15863.5 & 16121 & 1.60 & tlim & 15863.5 & 15917 & 0.34 & tlim & 15863.5 & 16121 & 1.60 & tlim & 15863.5 & 16121 & 1.60 & tlim \\
\hline & & 10 & 16282.5 & 16371 & 0.54 & tlim & 16282.5 & 16428 & 0.89 & tlim & 16282.5 & 16428 & 0.89 & tlim & 16282.5 & 16428 & 0.89 & tlim \\
\hline Avg. & & & 16280.3 & 16420.2 & 0.87 & 7200.0 & 16280.3 & 16391.6 & 0.68 & 7200.0 & 16280.3 & 16437.5 & 0.99 & 6998.1 & 16280.3 & 16450.8 & 1.05 & 7200.0 \\
\hline
\end{tabular}

seems to be worth in several cases. These results are even more promising when tackling problem instances considering a traffic of 150 vessels. As can be seen in Table 8, the additional time required to use the variable-fixing method is worth-while in most cases. Furthermore, in Table 8 most of the optimal solutions and best upper bounds are reported by using this approach.

Table 9 summarizes the previous results in this work while indicating the best performing model for each instance set. Thus, the table reports, for all nine methods and for each group of benchmark instances from the literature, the number of instances solved to proven optimality, opt, and the average execution time, $t(s)$. Column gap(\%) reports the average percentage gap with respect to the best lower bound obtained per each instance. For the benchmark instances of Cordeau et al. (2005) and Lalla-Ruiz et al. (2012), all instances are solved to optimality by all nine methods. Therefore, it can be seen that our reduced-cost-based variable-fixing method on top of $\mathrm{TI}$ and $\mathrm{AF}$ provides the smallest average execution times. Concerning the large-size instances of Nishi et al. (2016), $\mathrm{AF}_{+}^{\mathrm{rc}}$ solves more instances among all methods (46 out of
50), with the smallest average execution time and with the best quality upper bounds. In general words, our experiments indicate that $\mathrm{AF}_{+}^{\mathrm{rc}}$ performed better than all other methods. It is worth noting that the remaining 4 instances were solved to proven optimality by Nishi et al. (2016) in large computing times. The authors proved optimality for 9 out of 10 instances with 150 vessels by running the GSPP formulation for approximately 25, 000 seconds on average. The only open instance left by Nishi et al. (2016), i.e., instance $150 \times 15-08$, has been solved to proven optimality by our enhanced models in less than two hours, so all optimal solutions are now assessed for these benchmarks. That led us to create the new set of very large instances that is evaluated in the next section.

\subsection{Computational results on the new set of instances}

With the aim of studying the performance of our approaches in larger scenarios, a new set of problem instances is proposed. This new set was generated following the scheme provided in 
Cordeau et al. (2005). We considered values of the pair $(n, m)$ in $\{(200,15) ;(250,20)\}$, and for each pair we generated 10 instances, obtaining a total of 20 new instances. Table 10 shows a summary of the size of the models in terms of average number of variables (cols) and constraints (rows) reported in thousands.

Table 11 presents the results for the instances with 200 vessels and 15 berths, while Table 12 does the same for the instances with 250 vessels and 20 berths. In those tables, columns labeled as $l b, u b, g a p(\%)$ and $t(s)$ indicate, for each instance and method, the final lower and upper bounds, the percentage gap, and the computational time, respectively. Note that we do not report results for GSPP, GSPP ${ }_{+}, \mathrm{GSPP}_{+}^{\mathrm{rc}}$, TI and AF. The methods based on the GSPP formulation were not able to solve any of the new instances due to memory limit that can be inputed to the need of storing matrices $A$ and $B$. Concerning $\mathrm{TI}$ and $\mathrm{AF}$, we decided not to test them as they are outperformed by $\mathrm{TI}_{+}$and $\mathrm{AF}_{+}$, as indicated in the previous experiments reported in Tables 4 and 5 .

For the instances with 200 vessels, it can be observed that the methods considering the reduced-cost variable-fixing preprocessing tend to be, on average, faster than their versions without such preprocessing. Among the 10 instances of this group, the approaches based on the TI formulation were able to solve to optimality 3 problem instances, while 4 instances were solved to optimality by the methods based on AF. Slight improvements can be noticed in the lower bound values when the reduced-cost variablefixing technique is applied.

With regards to the instances with 250 vessels, only 1 out of 10 could be solved to proven optimality, i.e., instance $250 \times 20-06$ by $\mathrm{AF}_{+}$. For all other instances, the time limit of 2 hours was reached without proof of optimality. Analyzing these results, it can be seen that for very large instances using AF faces difficulties in improving the given initial upper bounds, differently from using TI which resulted in improving some of them. In terms of best solutions, $\mathrm{TI}_{+}^{\mathrm{rc}}$ is superior to the other methods because it provides 7 best solutions while $\mathrm{TI}_{+}, \mathrm{AF}_{+}$and $\mathrm{AF}_{+}^{\mathrm{rc}}$ provide 5, 4 and 3, respectively. In terms of percentage gaps, all methods were able to obtain low average gaps.

In general, the results in Tables 11 and 12 show that the TI based formulations perform slightly better than the AF ones on larger instances. This result can be explained by the fact that AF formulations make use of additional variables, thus resulting in a model with more variables than the TI models, as shown in Table 10. The use of these additional variables seems to impact the performance of AF models at certain sizes as those defined on the large problem instances.

\section{Conclusions}

In this work, we have addressed the dynamic berth allocation problem (DBAP) from a mathematical modeling perspective by providing and assessing two novel time-indexed (TI) and arc-flow (AF) formulations. We have also proposed modeling enhancements aimed at grouping similar berths and vessels, and a variable-fixing procedure based on reduced costs. Extensive computational experiments on benchmark instances have been performed to evaluate the investigated methods and compare them with the best model from the literature, that is, the generalized set-partitioning problem (GSPP) formulation.
The AF formulation performs better than the TI, which in turn performs better than the GSPP. Therefore, AF is advisable when used as a standalone model. The proposed modeling enhancements based on grouping similar vessels and berths improved the performance of all models by reducing the number of variables and leading to better computational times. The reduced-cost variable-fixing procedure leads to further improvements for all formulations. In particular, by applying this procedure to the AF model we could solve to proven optimality 166 out of 170 benchmark instances from the literature within two hours of time limit, including the only instance that was still open. Based on these findings, the AF formulation has shown superiority compared to the other formulations, and thus, it is recommended to be used in real-world environments. Taking into account the good results on the benchmark instances, a new set containing 20 large-sized instances involving between 200 and 250 vessels has been proposed. The GSPP models were not able to deal with these new instances due to excessive memory requirements. On the contrary, models based on TI and AF did not show memory problems and managed to optimally solve 5 instances and provide small gaps for the other ones.

As future work, we plan to adapt these novel formulations to other maritime logistics problems where the berth allocation is involved. In this sense, the joint consideration of the quay crane allocation and scheduling problem with berth scheduling, such as the one in Agra and Oliveira (2018), and continuous berth allocation problems (see e.g. Frojan, Correcher, Álvarez-Valdés, Koulouris, \& Tamarit, 2015), appear to be interesting future research directions.

\section{Acknowledgments}

This research was partially funded by the CNPq - Conselho Nacional de DesenvolvimentoCientífico e Tecnológico, Brazil, grant No. 234814/2014-4 and by the Spanish Ministry of Economy and Competitiveness (projects TIN2015-70226-R) and by MIUR-Italy (Grant PRIN 2015, Nonlinear and Combinatorial Aspects of Complex Networks).

\section{Appendix A. Detailed results}

In this appendix, we report detailed results obtained by all the mathematical programming approaches studied in the paper. Table A.13 details the results for the set of 90 instances proposed by Lalla-Ruiz et al. (2012). The results for the 30 instances proposed by Cordeau et al. (2005) are shown in Table A.14, while Table A.15 reports the results for the 50 benchmark instances of Nishi et al. (2016). Finally, Table A.16 contains the results for the set of 20 new instances proposed in this work. In all tables, we report the best lower (column $l b$ ) and upper (column $u b$ ) bounds found for each instance (considering all mathematical programming approaches). For each method and instance columns $t(s)$ and nd present the total execution time in seconds and the number of explored nodes, respectively. In addition, for each instance, the results (upper bound and execution time) obtained by the metaheuristic by Lalla-Ruiz et al. (2016) are shown under the column $\mathrm{MH}$. It has to be noted that these times are already considered in $t(s)$ of the reduced-cost approach. The last line of each table reports average values. 
Table A.13

Detailed results for the Lalla-Ruiz et al. (2012) benchmark instances

\begin{tabular}{|c|c|c|c|c|c|c|c|c|c|c|c|c|c|c|c|c|c|c|c|c|c|c|c|c|}
\hline$n$ & $m$ & id & $\mathrm{lb}$ & $\mathrm{ub}$ & Genera & lized & set par & tition & ing & & Time $\mathrm{i}$ & ndexed & & & & & Arc-flc & & & & & & $\mathrm{MH}$ & \\
\hline & & & & & GSPP & & GSPP & & GSPP & & $\mathrm{TI}$ & & $\mathrm{TI}_{+}$ & & $\mathrm{TI}_{+}^{\mathrm{rc}}$ & & $\mathrm{AF}$ & & $\mathrm{AF}_{+}$ & & $\mathrm{AF}_{+}^{\mathrm{rc}}$ & & & \\
\hline & & & & & $\mathrm{t}(\mathrm{s})$ & nd & $\mathrm{t}(\mathrm{s})$ & nd & $\mathrm{t}(\mathrm{s})$ & nd & $\mathrm{t}(\mathrm{s})$ & nd & $\mathrm{t}(\mathrm{s})$ & nd & $\mathrm{t}(\mathrm{s})$ & nd & $\mathrm{t}(\mathrm{s})$ & nd & $\mathrm{t}(\mathrm{s})$ & nd & $\mathrm{t}(\mathrm{s})$ & nd & $\mathrm{t}(\mathrm{s})$ & $\mathrm{ub}$ \\
\hline 30 & 3 & 01 & 1763 & 1763 & 10.4 & 0 & 7.2 & 0 & 5.6 & 0 & 10.5 & 0 & 5.5 & 0 & 1.8 & 0 & 20.3 & 178 & 12.1 & 133 & 0.8 & 0 & 0.2 & 1763 \\
\hline & & 02 & 2090 & 2090 & 17.0 & 9 & 11.5 & 86 & 9.0 & 5 & 17.5 & 22 & 13.2 & 23 & 3.9 & 12 & 11.1 & 654 & 15.6 & 648 & 4.5 & 291 & 0.4 & 2090 \\
\hline & & 03 & 2186 & 2186 & 7.3 & 0 & 6.6 & 0 & 6.2 & 0 & 7.4 & 0 & 9.6 & 0 & 1.8 & 0 & 7.7 & 61 & 3.7 & 0 & 0.9 & 0 & 0.4 & 2186 \\
\hline & & 04 & 1538 & 1538 & 8.1 & 0 & 7.2 & 0 & 5.6 & 0 & 4.6 & 0 & 5.2 & 0 & 2.0 & 0 & 6.6 & 37 & 4.9 & 15 & 1.1 & 0 & 0.3 & 1538 \\
\hline & & 05 & 2114 & 2114 & 15.6 & 0 & 14.0 & 13 & 7.6 & 11 & 23.2 & 11 & 10.4 & 53 & 4.1 & 9 & 10.3 & 60 & 5.8 & 75 & 1.8 & 11 & 0.1 & 2114 \\
\hline & & 06 & 2185 & 2185 & 12.2 & 0 & 15.4 & 0 & 6.6 & 0 & 14.1 & 0 & 15.3 & 0 & 2.7 & 0 & 95.5 & 1069 & 76.5 & 1486 & 10.9 & 702 & 0.1 & 2185 \\
\hline & & 07 & 1845 & 1845 & 16.7 & 50 & 12.9 & 46 & 9.4 & 53 & 18.7 & 65 & 16.3 & 130 & 5.0 & 36 & 20.8 & 221 & 12.3 & 205 & 10.2 & 1043 & 0.1 & 1845 \\
\hline & & 08 & 1271 & 1271 & 7.8 & 28 & 5.7 & 12 & 5.3 & 50 & 7.6 & 9 & 6.8 & 35 & 1.1 & 0 & 4.9 & 22 & 4.4 & 222 & 0.8 & 0 & 0.1 & 1271 \\
\hline & & 09 & 1595 & 1595 & 14.7 & 760 & 10.9 & 273 & 9.5 & 204 & 15.4 & 428 & 12.0 & 245 & 2.8 & 78 & 10.9 & 171 & 15.6 & 748 & 10.6 & 808 & 0.1 & 1595 \\
\hline & & 10 & 2195 & 2195 & 11.2 & 0 & 6.6 & 0 & 7.3 & 0 & 11.0 & 0 & 3.1 & 0 & 1.8 & 0 & 2.4 & 0 & 2.2 & 0 & 0.8 & 0 & 0.3 & 2195 \\
\hline & 5 & 01 & 1149 & 1149 & 10.7 & 0 & 7.6 & 0 & 13.6 & 0 & 5.3 & 0 & 6.3 & 0 & 0.8 & 0 & 1.8 & 0 & 1.2 & 0 & 0.8 & 0 & 0.1 & 1149 \\
\hline & & 02 & 1475 & 1475 & 21.6 & 0 & 12.1 & 0 & 11.7 & 0 & 20.5 & 0 & 11.5 & 0 & 1.5 & 0 & 3.9 & 0 & 3.2 & 0 & 1.0 & 0 & 0.2 & 1476 \\
\hline & & 03 & 1542 & 1542 & 25.2 & 0 & 12.5 & 13 & 11.0 & 0 & 13.4 & 7 & 15.8 & 9 & 1.8 & 0 & 9.0 & 0 & 2.4 & 0 & 1.1 & 0 & 0.4 & 1542 \\
\hline & & 04 & 1075 & 1075 & 12.0 & 0 & 8.2 & 0 & 9.0 & 0 & 4.2 & 0 & 5.2 & 0 & 1.0 & 0 & 3.3 & 0 & 2.4 & 0 & 0.8 & 0 & 0.2 & 1075 \\
\hline & & 05 & 1463 & 1463 & 11.3 & 0 & 12.0 & 0 & 10.0 & 0 & 11.8 & 0 & 7.2 & 0 & 1.1 & 0 & 4.9 & 0 & 2.8 & 0 & 0.8 & 0 & 0.1 & 1463 \\
\hline & & 06 & 1580 & 1580 & 12.9 & 0 & 11.2 & 0 & 8.8 & 0 & 20.8 & 0 & 12.9 & 0 & 1.7 & 0 & 4.6 & 0 & 5.0 & 0 & 1.1 & 0 & 0.2 & 1580 \\
\hline & & 07 & 1276 & 1276 & 10.6 & 0 & 9.2 & 0 & 8.7 & 0 & 5.5 & 0 & 8.0 & 0 & 1.0 & 0 & 3.2 & 0 & 2.2 & 0 & 0.8 & 0 & 0.1 & 1276 \\
\hline & & 08 & 870 & 870 & 12.5 & 0 & 7.5 & 0 & 10.6 & 0 & 5.9 & 0 & 7.0 & 0 & 1.1 & 0 & 5.8 & 0 & 3.1 & 0 & 0.9 & 0 & 0.3 & 870 \\
\hline & & 09 & 1134 & 1134 & 13.1 & 0 & 12.0 & 0 & 10.1 & 0 & 9.4 & 0 & 10.6 & 0 & 1.2 & 0 & 6.2 & 11 & 3.5 & 0 & 0.9 & 0 & 0.1 & 1144 \\
\hline & & 10 & 1527 & 1527 & 14.0 & 0 & 9.3 & 0 & 10.5 & 0 & 9.8 & 0 & 9.2 & 0 & 1.4 & 0 & 5.4 & 0 & 3.5 & 0 & 1.0 & 0 & 0.2 & 1527 \\
\hline 40 & 5 & 01 & 2301 & 2301 & 34.0 & 0 & 11.2 & 0 & 9.5 & 0 & 29.5 & 0 & 6.6 & 0 & 2.3 & 0 & 6.3 & 0 & 2.0 & 0 & 1.0 & 0 & 0.3 & 2303 \\
\hline & & 02 & 2829 & 2829 & 43.2 & 33 & 15.7 & 15 & 10.0 & 0 & 57.4 & 83 & 18.0 & 0 & 3.6 & 0 & 18.3 & 49 & 5.7 & 58 & 1.4 & 10 & 0.3 & 2829 \\
\hline & & 03 & 2880 & 2880 & 63.5 & 187 & 24.0 & 12 & 12.6 & 0 & 99.0 & 190 & 18.6 & 9 & 4.2 & 7 & 91.1 & 458 & 51.4 & 2083 & 1.9 & 8 & 0.2 & 2881 \\
\hline & & 04 & 2001 & 2001 & 25.7 & 0 & 12.0 & 0 & 9.3 & 34 & 17.5 & 0 & 9.0 & 0 & 1.7 & 0 & 9.5 & 0 & 4.0 & 0 & 1.2 & 0 & 0.4 & 2001 \\
\hline & & 05 & 2815 & 2815 & 66.0 & 102 & 24.1 & 13 & 14.2 & 24 & 85.4 & 43 & 33.4 & 18 & 5.5 & 9 & 234.7 & 1033 & 9.8 & 112 & 3.0 & 41 & 0.4 & 2815 \\
\hline & & 06 & 2934 & 2934 & 66.6 & 27 & 24.4 & 9 & 15.6 & 29 & 72.8 & 19 & 21.4 & 16 & 6.3 & 24 & 195.9 & 1066 & 45.5 & 1033 & 3.0 & 69 & 0.6 & 2934 \\
\hline & & 07 & 2632 & 2632 & 40.0 & 0 & 13.7 & 0 & 9.3 & 0 & 20.6 & 0 & 15.5 & 0 & 2.3 & 0 & 5.7 & 0 & 3.5 & 0 & 1.0 & 0 & 0.2 & 2634 \\
\hline & & 08 & 1835 & 1835 & 36.3 & 0 & 12.7 & 0 & 8.6 & 0 & 37.3 & 50 & 9.7 & 0 & 1.9 & 0 & 24.7 & 212 & 5.8 & 16 & 1.4 & 11 & 0.3 & 1836 \\
\hline & & 09 & 2086 & 2086 & 36.0 & 18 & 11.3 & 7 & 10.6 & 8 & 31.2 & 9 & 13.1 & 7 & 2.8 & 7 & 37.7 & 119 & 3.5 & 0 & 2.0 & 11 & 0.2 & 2094 \\
\hline & & 10 & 2962 & 2962 & 27.0 & 0 & 15.8 & 0 & 9.7 & 0 & 47.3 & 14 & 14.3 & 0 & 3.8 & 0 & 7.8 & 0 & 6.4 & 0 & 1.3 & 0 & 0.4 & 2962 \\
\hline & 7 & 01 & 1458 & 1458 & 37.1 & 0 & 20.2 & 0 & 24.6 & 0 & 22.5 & 0 & 15.3 & 0 & 2.1 & 0 & 10.9 & 0 & 4.3 & 0 & 2.0 & 0 & 0.2 & 1464 \\
\hline & & 02 & 1375 & 1375 & 25.7 & 0 & 15.1 & 0 & 20.3 & 0 & 8.9 & 0 & 8.4 & 0 & 1.2 & 0 & 3.7 & 0 & 2.3 & 0 & 1.4 & 0 & 0.3 & 1378 \\
\hline & & 03 & 2119 & 2119 & 55.2 & 0 & 28.9 & 0 & 24.3 & 0 & 34.9 & 0 & 21.0 & 0 & 4.0 & 0 & 14.6 & 10 & 5.1 & 0 & 2.5 & 0 & 0.3 & 2134 \\
\hline & & 04 & 1591 & 1591 & 37.9 & 0 & 26.3 & 0 & 20.1 & 0 & 22.2 & 0 & 10.6 & 0 & 1.8 & 0 & 8.6 & 0 & 3.1 & 0 & 1.7 & 0 & 0.3 & 1601 \\
\hline & & 05 & 1847 & 1847 & 40.3 & 0 & 23.5 & 0 & 19.7 & 0 & 24.6 & 0 & 14.6 & 0 & 2.0 & 0 & 8.5 & 0 & 5.1 & 0 & 1.8 & 0 & 0.5 & 1849 \\
\hline & & 06 & 2080 & 2080 & 46.6 & 0 & 25.4 & 0 & 19.0 & 0 & 25.2 & 0 & 18.0 & 0 & 2.0 & 0 & 7.6 & 0 & 5.2 & 0 & 1.4 & 0 & 0.4 & 2080 \\
\hline & & 07 & 1841 & 1841 & 37.7 & 0 & 25.0 & 0 & 18.2 & 13 & 28.1 & 8 & 15.1 & 0 & 1.9 & 0 & 10.4 & 0 & 7.7 & 0 & 1.6 & 0 & 0.3 & 1842 \\
\hline & & 08 & 2025 & 2025 & 36.9 & 0 & 24.8 & 0 & 22.7 & 0 & 28.5 & 0 & 16.7 & 0 & 2.0 & 0 & 9.7 & 0 & 7.3 & 0 & 1.4 & 0 & 0.3 & 2025 \\
\hline & & 09 & 1880 & 1880 & 27.4 & 0 & 15.5 & 0 & 19.6 & 0 & 24.7 & 0 & 9.6 & 0 & 1.4 & 0 & 9.8 & 0 & 5.0 & 0 & 1.3 & 0 & 0.3 & 1880 \\
\hline & & 10 & 1883 & 1883 & 37.0 & 0 & 20.9 & 0 & 21.0 & 0 & 44.2 & 0 & 11.6 & 0 & 2.4 & 0 & 8.3 & 0 & 2.3 & 0 & 1.6 & 0 & 0.3 & 1890 \\
\hline 55 & 5 & 01 & 4689 & 4689 & 86.2 & 80 & 17.9 & 0 & 13.7 & 0 & 156.0 & 420 & 14.3 & 0 & 4.7 & 0 & 45.4 & 25 & 5.8 & 0 & 1.9 & 0 & 0.7 & 4689 \\
\hline & & 02 & 5467 & 5467 & 52.5 & 0 & 9.8 & 0 & 10.8 & 0 & 58.9 & 0 & 4.5 & 0 & 3.9 & 0 & 10.6 & 0 & 3.3 & 0 & 1.8 & 0 & 1.1 & 5467 \\
\hline & & 03 & 5499 & 5499 & 57.9 & 0 & 24.1 & 0 & 12.7 & 18 & 73.9 & 0 & 17.0 & 4 & 4.5 & 0 & 8.6 & 0 & 6.6 & 20 & 1.9 & 0 & 1.1 & 5499 \\
\hline & & 04 & 4165 & 4165 & 67.9 & 0 & 14.7 & 0 & 11.1 & 0 & 48.8 & 0 & 9.9 & 0 & 3.9 & 0 & 51.6 & 39 & 11.3 & 394 & 1.6 & 0 & 0.6 & 4165 \\
\hline & & 05 & 5478 & 5478 & 55.8 & 0 & 16.7 & 0 & 11.0 & 0 & 46.7 & 0 & 14.3 & 0 & 3.9 & 0 & 21.4 & 13 & 3.8 & 0 & 1.4 & 0 & 0.6 & 5478 \\
\hline & & 06 & 5595 & 5595 & 63.7 & 0 & 17.5 & 0 & 11.1 & 0 & 56.9 & 0 & 4.2 & 0 & 4.3 & 0 & 10.0 & 0 & 3.4 & 0 & 1.6 & 0 & 0.8 & 5597 \\
\hline & & 07 & 4870 & 4870 & 27.1 & 0 & 8.6 & 0 & 10.7 & 0 & 17.5 & 0 & 4.5 & 0 & 3.3 & 0 & 13.9 & 5 & 2.6 & 0 & 1.5 & 0 & 0.6 & 4878 \\
\hline & & 08 & 3552 & 3552 & 145.9 & 147 & 27.7 & 60 & 12.9 & 20 & 143.5 & 194 & 29.4 & 33 & 6.5 & 11 & 57.2 & 33 & 59.4 & 971 & 2.8 & 31 & 0.8 & 3552 \\
\hline & & 09 & 4273 & 4273 & 54.6 & 0 & 8.8 & 0 & 10.9 & 0 & 33.6 & 0 & 12.3 & 0 & 3.7 & 0 & 8.8 & 0 & 10.1 & 66 & 1.7 & 0 & 0.6 & 4277 \\
\hline & & 10 & 5739 & 5739 & 36.1 & 0 & 7.5 & 0 & 10.5 & 0 & 55.0 & 0 & 14.4 & 0 & 3.3 & 0 & 23.9 & 14 & 4.2 & 0 & 1.3 & 0 & 0.6 & 5739 \\
\hline & 7 & 01 & 2846 & 2846 & 97.9 & 0 & 32.2 & 0 & 22.5 & 0 & 83.9 & 70 & 11.0 & 0 & 3.3 & 0 & 25.0 & 29 & 10.6 & 13 & 2.1 & 0 & 0.6 & 2846 \\
\hline & & 02 & 2883 & 2883 & 124.4 & 109 & 25.0 & 8 & 25.1 & 0 & 108.4 & 13 & 15.2 & 0 & 10.3 & 0 & 30.4 & 29 & 4.8 & 0 & 4.6 & 10 & 0.6 & 2894 \\
\hline & & 03 & 3825 & 3825 & 60.8 & 0 & 31.8 & 0 & 23.8 & 0 & 47.2 & 0 & 7.6 & 0 & 4.1 & 0 & 15.5 & 0 & 5.8 & 0 & 1.7 & 0 & 0.6 & 3844 \\
\hline & & 04 & 2951 & 2951 & 60.6 & 0 & 19.5 & 0 & 21.5 & 0 & 35.7 & 0 & 6.8 & 0 & 2.8 & 0 & 11.4 & 0 & 5.0 & 0 & 1.9 & 0 & 0.6 & 2967 \\
\hline & & 05 & 3797 & 3797 & 94.8 & 99 & 24.6 & 25 & 26.7 & 20 & 54.1 & 20 & 26.9 & 9 & 5.3 & 0 & 52.8 & 49 & 12.3 & 72 & 2.6 & 0 & 0.5 & 3803 \\
\hline & & 06 & 3783 & 3783 & 79.6 & 0 & 36.1 & 0 & 21.7 & 0 & 53.3 & 0 & 19.0 & 0 & 4.4 & 8 & 24.9 & 22 & 9.0 & 20 & 2.3 & 12 & 0.7 & 3783 \\
\hline & & 07 & 3774 & 3774 & 62.5 & 0 & 18.9 & 0 & 19.6 & 0 & 44.9 & 0 & 15.3 & 0 & 4.1 & 0 & 11.7 & 0 & 7.5 & 0 & 2.1 & 0 & 0.6 & 3774 \\
\hline & & 08 & 3862 & 3862 & 119.7 & 60 & 28.2 & 0 & 23.6 & 0 & 89.4 & 16 & 16.8 & 0 & 5.1 & 0 & 37.0 & 50 & 7.8 & 0 & 1.9 & 0 & 0.6 & 3862 \\
\hline & & 09 & 3591 & 3591 & 129.3 & 998 & 38.2 & 0 & 21.0 & 0 & 95.2 & 66 & 31.6 & 12 & 6.5 & 49 & 42.8 & 77 & 14.0 & 122 & 11.5 & 1587 & 0.6 & 3597 \\
\hline & & 10 & 3623 & 3623 & 208.6 & 404 & 36.7 & 156 & 32.3 & 334 & 456.4 & 1889 & 30.6 & 181 & 11.8 & 83 & 313.4 & 1198 & 63.1 & 1124 & 5.2 & 79 & 0.5 & 3654 \\
\hline & 10 & 01 & 2742 & 2742 & 81.3 & 0 & 18.3 & 0 & 28.3 & 0 & 44.4 & 0 & 6.2 & 0 & 3.5 & 0 & 15.9 & 0 & 4.2 & 0 & 2.7 & 0 & 0.8 & 2754 \\
\hline & & 02 & 2527 & 2527 & 144.9 & 0 & 23.7 & 0 & 25.1 & 0 & 56.4 & 0 & 13.0 & 0 & 4.1 & 0 & 67.9 & 86 & 8.3 & 0 & 3.4 & 11 & 0.6 & 2531 \\
\hline & & 03 & 2544 & 2544 & 98.7 & 0 & 19.7 & 0 & 26.7 & 0 & 23.8 & 0 & 10.1 & 0 & 2.5 & 0 & 15.8 & 0 & 4.0 & 0 & 2.5 & 0 & 0.7 & 2547 \\
\hline & & 04 & 3315 & 3315 & 135.0 & 0 & 25.9 & 0 & 26.1 & 0 & 48.9 & 0 & 9.6 & 0 & 2.9 & 0 & 33.2 & 4 & 5.7 & 0 & 2.2 & 0 & 0.7 & 3315 \\
\hline & & 05 & 3109 & 3109 & 162.5 & 0 & 28.3 & 0 & 27.7 & 0 & 58.2 & 0 & 13.7 & 0 & 4.4 & 0 & 25.7 & 0 & 8.6 & 0 & 2.8 & 0 & 0.6 & 3118 \\
\hline & & 06 & 2283 & 2283 & 79.0 & 0 & 18.8 & 0 & 26.1 & 0 & 16.0 & 0 & 4.1 & 0 & 1.5 & 0 & 14.1 & 0 & 2.8 & 0 & 1.7 & 0 & 0.6 & 2283 \\
\hline & & 07 & 2144 & 2144 & 89.6 & 0 & 19.3 & 0 & 27.5 & 0 & 45.9 & 0 & 8.8 & 0 & 3.5 & 0 & 39.7 & 107 & 5.8 & 0 & 3.1 & 0 & 0.7 & 2150 \\
\hline & & 08 & 2720 & 2720 & 95.6 & 0 & 26.0 & 29 & 26.7 & 0 & 85.3 & 86 & 16.9 & 0 & 6.5 & 990 & 66.4 & 92 & 9.8 & 0 & 2.7 & 0 & 0.9 & 2723 \\
\hline & & 09 & 2149 & 2149 & 73.0 & 0 & 18.4 & 0 & 27.1 & 0 & 33.8 & 0 & 4.4 & 0 & 2.1 & 0 & 15.5 & 0 & 5.2 & 0 & 2.1 & 0 & 0.8 & 2162 \\
\hline & & 10 & 2814 & 2814 & 146.4 & 0 & 25.5 & 0 & 28.1 & 0 & 72.5 & 0 & 13.0 & 0 & 2.5 & 0 & 18.1 & 0 & 8.2 & 0 & 2.2 & 0 & 0.7 & 2814 \\
\hline
\end{tabular}


Table A.13 (continued)

\begin{tabular}{|c|c|c|c|c|c|c|c|c|c|c|c|c|c|c|c|c|c|c|c|c|c|c|c|c|}
\hline \multirow[t]{3}{*}{$n$} & \multirow[t]{3}{*}{$m$} & \multirow[t]{3}{*}{ id } & \multirow[t]{3}{*}{$\mathrm{lb}$} & \multirow[t]{3}{*}{ ub } & \multicolumn{6}{|c|}{ Generalized set partitioning } & \multicolumn{6}{|c|}{ Time indexed } & \multicolumn{6}{|c|}{ Arc-flow } & \multicolumn{2}{|l|}{$\mathrm{MH}$} \\
\hline & & & & & \multicolumn{2}{|l|}{ GSPP } & \multicolumn{2}{|c|}{$\mathrm{GSPP}_{+}$} & \multicolumn{2}{|c|}{$\mathrm{GSPP}_{+}^{\mathrm{rc}}$} & \multicolumn{2}{|l|}{$\mathrm{TI}$} & \multicolumn{2}{|l|}{$\mathrm{TI}_{+}$} & \multicolumn{2}{|l|}{$\mathrm{TI}_{+}^{\mathrm{rc}}$} & \multicolumn{2}{|l|}{$\mathrm{AF}$} & \multicolumn{2}{|l|}{$\mathrm{AF}_{+}$} & \multicolumn{2}{|l|}{$\mathrm{AF}_{+}^{\mathrm{rc}}$} & \multirow[b]{2}{*}{$t(s)$} & \multirow[b]{2}{*}{ ub } \\
\hline & & & & & $\mathrm{t}(\mathrm{s})$ & nd & $\mathrm{t}(\mathrm{s})$ & nd & $\mathrm{t}(\mathrm{s})$ & nd & $\mathrm{t}(\mathrm{s})$ & nd & $t(s)$ & nd & $\mathrm{t}(\mathrm{s})$ & nd & $t(s)$ & nd & $\mathrm{t}(\mathrm{s})$ & nd & $\mathrm{t}(\mathrm{s})$ & nd & & \\
\hline \multirow[t]{20}{*}{60} & \multirow[t]{10}{*}{5} & 01 & 5753 & 5753 & 45.6 & 0 & 21.3 & 0 & 11.2 & 0 & 62.4 & 0 & 9.7 & 0 & 5.0 & 0 & 42.5 & 41 & 3.8 & 0 & 1.5 & 0 & 0.9 & 5753 \\
\hline & & 02 & 6884 & 6884 & 308.7 & 573 & 55.3 & 1470 & 57.3 & 2008 & 284.0 & 1049 & 55.2 & 1651 & 23.9 & 472 & 392.5 & 2762 & 80.9 & 985 & 8.6 & 918 & 0.6 & 6884 \\
\hline & & 03 & 6780 & 6780 & 65.3 & 0 & 17.8 & 0 & 11.0 & 0 & 39.8 & 0 & 4.5 & 0 & 3.3 & 0 & 23.4 & 27 & 3.8 & 0 & 1.3 & 0 & 0.7 & 6780 \\
\hline & & 04 & 5092 & 5092 & 110.3 & 19 & 14.3 & 26 & 10.2 & 0 & 54.4 & 0 & 10.3 & 0 & 4.4 & 0 & 53.0 & 47 & 3.9 & 0 & 1.9 & 0 & 0.8 & 5092 \\
\hline & & 05 & 6715 & 6715 & 83.5 & 0 & 19.7 & 0 & 12.8 & 0 & 78.2 & 0 & 15.4 & 0 & 5.4 & 0 & 27.5 & 10 & 3.6 & 0 & 1.6 & 0 & 0.8 & 6715 \\
\hline & & 06 & 6616 & 6616 & 75.3 & 0 & 7.1 & 0 & 10.7 & 0 & 37.5 & 0 & 8.5 & 0 & 5.3 & 0 & 44.0 & 19 & 3.1 & 0 & 1.3 & 0 & 0.7 & 6616 \\
\hline & & 07 & 6011 & 6011 & 59.6 & 0 & 20.2 & 0 & 10.5 & 0 & 36.0 & 0 & 4.7 & 0 & 4.5 & 0 & 23.5 & 6 & 4.8 & 0 & 1.4 & 0 & 0.7 & 6011 \\
\hline & & 08 & 4385 & 4385 & 128.6 & 101 & 26.0 & 11 & 11.6 & 10 & 77.6 & 24 & 30.6 & 13 & 4.4 & 0 & 25.5 & 21 & 44.9 & 2438 & 2.4 & 13 & 0.7 & 4385 \\
\hline & & 09 & 5235 & 5235 & 34.2 & 0 & 17.5 & 0 & 11.1 & 0 & 54.2 & 0 & 19.9 & 0 & 4.5 & 0 & 24.9 & 17 & 7.4 & 26 & 1.8 & 0 & 0.7 & 5239 \\
\hline & & 10 & 7255 & 7255 & 88.9 & 0 & 16.7 & 0 & 11.7 & 0 & 89.8 & 0 & 11.2 & 0 & 5.7 & 0 & 25.7 & 8 & 3.9 & 0 & 1.4 & 0 & 0.7 & 7255 \\
\hline & \multirow[t]{10}{*}{7} & 01 & 3707 & 3707 & 118.8 & 5 & 88.1 & 1333 & 61.0 & 1271 & 132.0 & 60 & 105.0 & 1772 & 28.2 & 0 & 34.4 & 49 & 22.9 & 86 & 7.6 & 28 & 1.2 & 3725 \\
\hline & & 02 & 4146 & 4146 & 426.4 & 1290 & 101.6 & 555 & 96.9 & 1222 & 505.3 & 2032 & 104.2 & 1387 & 98.8 & 1893 & 126.8 & 223 & 160.1 & 1643 & 18.8 & 331 & 0.7 & 4168 \\
\hline & & 03 & 4273 & 4273 & 301.2 & 783 & 78.8 & 183 & 59.7 & 133 & 273.3 & 363 & 83.8 & 503 & 33.0 & 220 & 111.1 & 156 & 53.6 & 367 & 31.1 & 988 & 0.9 & 4290 \\
\hline & & 04 & 3910 & 3910 & 120.7 & 0 & 50.8 & 0 & 38.8 & 0 & 106.2 & 92 & 37.0 & 0 & 14.5 & 0 & 76.7 & 37 & 40.0 & 206 & 4.0 & 0 & 0.9 & 3915 \\
\hline & & 05 & 4251 & 4251 & 238.1 & 1088 & 70.0 & 60 & 40.7 & 76 & 120.0 & 136 & 77.7 & 127 & 15.7 & 106 & 69.6 & 71 & 59.7 & 289 & 4.9 & 35 & 0.8 & 4253 \\
\hline & & 06 & 5727 & 5727 & 155.8 & 0 & 56.0 & 0 & 40.1 & 0 & 149.8 & 60 & 48.0 & 78 & 11.0 & 0 & 58.1 & 59 & 17.9 & 28 & 2.1 & 0 & 0.6 & 5727 \\
\hline & & 07 & 3719 & 3719 & 97.1 & 0 & 36.3 & 0 & 46.4 & 0 & 121.7 & 5 & 38.7 & 0 & 13.9 & 0 & 61.9 & 23 & 12.8 & 8 & 3.8 & 0 & 0.8 & 3744 \\
\hline & & 08 & 4582 & 4582 & 409.0 & 1062 & 125.5 & 635 & 193.8 & 1848 & 566.5 & 1892 & 287.7 & 2611 & 202.3 & 2610 & 983.4 & 1390 & 218.6 & 965 & 92.8 & 1283 & 0.7 & 4600 \\
\hline & & 09 & 3979 & 3979 & 123.8 & 11 & 46.1 & 4 & 78.4 & 320 & 264.7 & 200 & 79.6 & 75 & 17.3 & 39 & 143.2 & 101 & 116.6 & 466 & 41.0 & 1413 & 0.7 & 3994 \\
\hline & & 10 & 4107 & 4107 & 216.6 & 169 & 58.0 & 93 & 57.1 & 114 & 165.3 & 203 & 55.1 & 83 & 23.2 & 131 & 84.5 & 40 & 91.8 & 522 & 57.4 & 966 & 0.9 & 4121 \\
\hline \multicolumn{3}{|c|}{ Sum/Avg. } & - & - & 78.7 & 91 & 24.3 & 57 & 22.1 & 87 & 71.8 & 109 & 22.0 & 101 & 8.4 & 75 & 50.3 & 138 & 18.2 & 196 & 5.0 & 119 & 0.5 & - \\
\hline
\end{tabular}

Table A.14

Detailed results for the Cordeau et al. (2005) benchmark instances.

\begin{tabular}{|c|c|c|c|c|c|c|c|c|c|c|c|c|c|c|c|c|c|c|c|c|c|c|c|c|}
\hline \multirow[t]{3}{*}{$n$} & \multirow[t]{3}{*}{$m$} & \multirow[t]{3}{*}{ id } & \multirow[t]{3}{*}{$\mathrm{lb}$} & \multirow[t]{3}{*}{ ub } & \multicolumn{6}{|c|}{ Generalized set partitioning } & \multicolumn{6}{|c|}{ Time indexed } & \multicolumn{6}{|c|}{ Arc-flow } & \multicolumn{2}{|l|}{$\mathrm{MH}$} \\
\hline & & & & & \multicolumn{2}{|c|}{ GSPP } & \multicolumn{2}{|c|}{$\mathrm{GSPP}_{+}$} & \multicolumn{2}{|c|}{$\mathrm{GSPP}_{+}^{\mathrm{rc}}$} & \multicolumn{2}{|l|}{ TI } & \multicolumn{2}{|l|}{$\mathrm{TI}_{+}$} & \multicolumn{2}{|l|}{$\mathrm{TI}_{+}^{\mathrm{rc}}$} & \multicolumn{2}{|l|}{$\mathrm{AF}$} & \multicolumn{2}{|l|}{$\mathrm{AF}_{+}$} & \multicolumn{2}{|l|}{$\mathrm{AF}_{+}^{\mathrm{rc}}$} & \multirow[b]{2}{*}{$\mathrm{t}(\mathrm{s})$} & \multirow[b]{2}{*}{$\mathrm{ub}$} \\
\hline & & & & & $\mathrm{t}(\mathrm{s})$ & nd & $\mathrm{t}(\mathrm{s})$ & nd & $\mathrm{t}(\mathrm{s})$ & nd & $\mathrm{t}(\mathrm{s})$ & nd & $\mathrm{t}(\mathrm{s})$ & nd & $\mathrm{t}(\mathrm{s})$ & nd & $\mathrm{t}(\mathrm{s})$ & nd & $\mathrm{t}(\mathrm{s})$ & nd & $\mathrm{t}(\mathrm{s})$ & nd & & \\
\hline 60 & 13 & 01 & 1409 & 1409 & 13.7 & 0 & 1.9 & 0 & 3.7 & 0 & 3.7 & 0 & 2.9 & 0 & 1.6 & 0 & 6.4 & 0 & 1.1 & 0 & 1.8 & 0 & 1.5 & 1409 \\
\hline & & 02 & 1261 & 1261 & 11.6 & 0 & 1.9 & 0 & 3.9 & 0 & 2.9 & 0 & 0.7 & 0 & 1.8 & 0 & 4.6 & 0 & 1.0 & 0 & 2.0 & 0 & 1.7 & 1261 \\
\hline & & 03 & 1129 & 1129 & 11.4 & 0 & 1.7 & 0 & 3.4 & 0 & 2.6 & 0 & 0.6 & 0 & 1.3 & 0 & 3.5 & 0 & 1.0 & 0 & 1.5 & 0 & 1.1 & 1129 \\
\hline & & 04 & 1302 & 1302 & 12.0 & 0 & 1.8 & 0 & 3.9 & 0 & 3.2 & 0 & 0.7 & 0 & 1.8 & 0 & 2.4 & 0 & 0.9 & 0 & 2.0 & 0 & 1.6 & 1302 \\
\hline & & 05 & 1207 & 1207 & 12.2 & 0 & 1.9 & 0 & 4.1 & 0 & 2.8 & 0 & 0.6 & 0 & 1.8 & 0 & 3.5 & 0 & 0.6 & 0 & 2.0 & 0 & 1.6 & 1207 \\
\hline & & 06 & 1261 & 1261 & 13.0 & 0 & 1.8 & 0 & 3.4 & 0 & 2.9 & 0 & 1.9 & 0 & 1.4 & 0 & 2.4 & 0 & 1.5 & 0 & 1.6 & 0 & 1.2 & 1261 \\
\hline & & 07 & 1279 & 1279 & 11.8 & 0 & 2.0 & 0 & 3.8 & 0 & 2.9 & 0 & 0.7 & 0 & 1.7 & 0 & 2.4 & 0 & 1.0 & 0 & 2.0 & 0 & 1.6 & 1279 \\
\hline & & 08 & 1299 & 1299 & 12.5 & 0 & 1.9 & 0 & 4.2 & 0 & 3.2 & 0 & 0.7 & 0 & 2.0 & 0 & 3.9 & 0 & 0.6 & 0 & 2.2 & 0 & 1.8 & 1299 \\
\hline & & 09 & 1444 & 1444 & 12.4 & 0 & 2.0 & 0 & 4.2 & 0 & 3.2 & 0 & 0.8 & 0 & 1.9 & 0 & 3.8 & 0 & 0.6 & 0 & 2.2 & 0 & 1.7 & 1444 \\
\hline & & 10 & 1213 & 1213 & 12.3 & 0 & 1.8 & 0 & 4.3 & 0 & 3.3 & 0 & 0.7 & 0 & 2.1 & 0 & 2.6 & 0 & 1.3 & 0 & 2.4 & 0 & 2.0 & 1213 \\
\hline & & 11 & 1368 & 1368 & 13.7 & 0 & 2.0 & 0 & 4.1 & 0 & 3.4 & 0 & 0.8 & 0 & 1.9 & 0 & 5.3 & 0 & 1.6 & 0 & 2.1 & 0 & 1.7 & 1368 \\
\hline & & 12 & 1325 & 1325 & 13.2 & 0 & 1.9 & 0 & 4.2 & 0 & 3.1 & 0 & 0.8 & 0 & 2.0 & 0 & 5.8 & 0 & 1.0 & 0 & 2.2 & 0 & 1.8 & 1325 \\
\hline & & 13 & 1360 & 1360 & 12.1 & 0 & 1.9 & 0 & 4.1 & 0 & 3.2 & 0 & 0.8 & 0 & 1.9 & 0 & 4.5 & 0 & 1.3 & 0 & 2.1 & 0 & 1.7 & 1360 \\
\hline & & 14 & 1233 & 1233 & 13.0 & 0 & 1.9 & 0 & 4.1 & 0 & 3.8 & 0 & 0.7 & 0 & 1.7 & 0 & 5.7 & 0 & 1.1 & 0 & 2.0 & 0 & 1.6 & 1233 \\
\hline & & 15 & 1295 & 1295 & 12.7 & 0 & 1.9 & 0 & 4.0 & 0 & 3.1 & 0 & 1.6 & 0 & 1.8 & 0 & 5.1 & 0 & 0.6 & 0 & 2.0 & 0 & 1.6 & 1295 \\
\hline & & 16 & 1364 & 1364 & 12.2 & 0 & 1.9 & 0 & 4.3 & 0 & 4.0 & 0 & 0.7 & 0 & 2.1 & 0 & 4.3 & 0 & 1.1 & 0 & 2.3 & 0 & 1.9 & 1364 \\
\hline & & 17 & 1283 & 1283 & 12.6 & 0 & 1.8 & 0 & 3.8 & 0 & 2.9 & 0 & 1.3 & 0 & 1.6 & 0 & 4.8 & 0 & 0.6 & 0 & 1.8 & 0 & 1.4 & 1283 \\
\hline & & 18 & 1345 & 1345 & 12.1 & 0 & 2.0 & 0 & 4.0 & 0 & 3.1 & 0 & 1.2 & 0 & 1.8 & 0 & 5.1 & 0 & 0.6 & 0 & 2.0 & 0 & 1.7 & 1345 \\
\hline & & 19 & 1367 & 1367 & 11.9 & 0 & 2.0 & 0 & 3.8 & 0 & 3.5 & 0 & 0.8 & 0 & 1.7 & 0 & 3.5 & 0 & 1.1 & 0 & 1.9 & 0 & 1.5 & 1368 \\
\hline & & 20 & 1328 & 1328 & 13.8 & 0 & 2.2 & 0 & 3.8 & 0 & 5.7 & 0 & 1.1 & 0 & 1.5 & 0 & 6.0 & 0 & 0.6 & 0 & 1.7 & 0 & 1.3 & 1328 \\
\hline & & 21 & 1341 & 1341 & 12.4 & 0 & 1.9 & 0 & 3.4 & 0 & 3.2 & 0 & 0.8 & 0 & 1.3 & 0 & 4.6 & 0 & 0.8 & 0 & 1.5 & 0 & 1.2 & 1341 \\
\hline & & 22 & 1326 & 1326 & 12.7 & 0 & 2.2 & 0 & 3.7 & 0 & 3.3 & 0 & 1.3 & 0 & 1.5 & 0 & 3.7 & 0 & 0.9 & 0 & 1.7 & 0 & 1.3 & 1326 \\
\hline & & 23 & 1266 & 1266 & 11.3 & 0 & 1.8 & 0 & 3.9 & 0 & 2.9 & 0 & 0.7 & 0 & 1.8 & 0 & 4.6 & 0 & 0.6 & 0 & 2.0 & 0 & 1.7 & 1266 \\
\hline & & 24 & 1260 & 1260 & 12.0 & 0 & 1.8 & 0 & 3.6 & 0 & 2.8 & 0 & 0.7 & 0 & 1.4 & 0 & 4.0 & 0 & 0.8 & 0 & 1.6 & 0 & 1.2 & 1260 \\
\hline & & 25 & 1376 & 1376 & 13.5 & 0 & 2.1 & 0 & 3.8 & 0 & 3.5 & 0 & 0.8 & 0 & 1.3 & 0 & 2.8 & 0 & 0.7 & 0 & 1.6 & 0 & 1.1 & 1376 \\
\hline & & 26 & 1318 & 1318 & 11.8 & 0 & 1.8 & 0 & 3.7 & 0 & 4.0 & 0 & 0.7 & 0 & 1.5 & 0 & 4.2 & 0 & 1.1 & 0 & 1.7 & 0 & 1.3 & 1318 \\
\hline & & 27 & 1261 & 1261 & 11.8 & 0 & 1.8 & 0 & 3.7 & 0 & 2.9 & 0 & 0.7 & 0 & 1.5 & 0 & 3.2 & 0 & 1.1 & 0 & 1.7 & 0 & 1.3 & 1261 \\
\hline & & 28 & 1359 & 1359 & 12.4 & 0 & 2.2 & 0 & 3.6 & 0 & 3.3 & 0 & 0.7 & 0 & 1.3 & 0 & 2.6 & 0 & 1.1 & 0 & 1.5 & 0 & 1.1 & 1359 \\
\hline & & 29 & 1280 & 1280 & 12.9 & 0 & 1.8 & 0 & 3.6 & 0 & 3.1 & 0 & 0.7 & 0 & 1.4 & 0 & 3.8 & 0 & 0.9 & 0 & 1.6 & 0 & 1.2 & 1280 \\
\hline & & 30 & 1344 & 1344 & 13.0 & 0 & 1.9 & 0 & 3.6 & 0 & 3.2 & 0 & 0.8 & 0 & 1.4 & 0 & 5.4 & 0 & 1.0 & 0 & 1.6 & 0 & 1.2 & 1344 \\
\hline Sun & lvg. & & - & - & 12.5 & 0 & 1.9 & 0 & 3.9 & 0 & 3.3 & 0 & 0.9 & 0 & 1.7 & 0 & 4.1 & 0 & 0.9 & 0 & 1.9 & 0 & 1.5 & - \\
\hline
\end{tabular}


Table A.15

Detailed results for the Nishi et al. (2016) benchmark instances.

\begin{tabular}{|c|c|c|c|c|c|c|c|c|c|c|c|c|c|c|c|c|c|c|c|c|c|c|c|}
\hline \multirow[t]{3}{*}{$n$} & \multirow[t]{3}{*}{$m$} & \multirow[t]{3}{*}{ id $\mathrm{lb}$} & \multirow[t]{3}{*}{ ub } & \multicolumn{6}{|c|}{ Generalized set partitioning } & \multicolumn{6}{|c|}{ Time indexed } & \multicolumn{6}{|c|}{ Arc-flow } & \multicolumn{2}{|l|}{$\mathrm{MH}$} \\
\hline & & & & \multicolumn{2}{|l|}{ GSPP } & \multicolumn{2}{|l|}{$\mathrm{GSPP}_{+}$} & \multicolumn{2}{|l|}{$\mathrm{GSPP}_{+}^{\mathrm{rc}}$} & TI & & $\mathrm{TI}_{+}$ & & $\mathrm{TI}_{+}^{\mathrm{rc}}$ & & $\mathrm{AF}$ & & $\mathrm{AF}_{+}$ & & $\mathrm{AF}_{+}^{\mathrm{rc}}$ & & & \\
\hline & & & & $t(s)$ & nd & $t(s)$ & nd & $t(s)$ & nd & $t(s)$ & nd & $\mathrm{t}(\mathrm{s})$ & nd & $t(s)$ & nd & $\mathrm{t}(\mathrm{s})$ & nd & $\mathrm{t}(\mathrm{s})$ & nd & $\mathrm{t}(\mathrm{s})$ & nd & $t(s)$ & $\mathrm{ub}$ \\
\hline 80 & 10 & 013417 & 3417 & 177.4 & 119 & 168.1 & 90 & 194.0 & 129 & 152.0 & 390 & 142.6 & 307 & 26.6 & 80 & 198.3 & 384 & 119.8 & 298 & 17.0 & 190 & 1.8 & 3427 \\
\hline & & 022602 & 2602 & 138.5 & 0 & 147.3 & 0 & 193.4 & 2 & 106.7 & 4 & 127.6 & 4 & 25.8 & 14 & 114.6 & 136 & 93.1 & 136 & 20.9 & 207 & 3.0 & 2637 \\
\hline & & 032602 & 2602 & 138.5 & 0 & 147.0 & 0 & 191.7 & 6 & 106.3 & 4 & 130.2 & 4 & 23.0 & 30 & 114.6 & 136 & 93.2 & 136 & 17.9 & 123 & 2.0 & 2628 \\
\hline & & 043504 & 3504 & 179.2 & 13 & 191.0 & 13 & 204.3 & 22 & 149.8 & 27 & 188.3 & 27 & 55.4 & 284 & 144.0 & 642 & 118.0 & 642 & 42.1 & 531 & 2.3 & 3530 \\
\hline & & 054010 & 4010 & 175.5 & 55 & 186.6 & 55 & 211.6 & 20 & 166.8 & 94 & 204.9 & 94 & 60.3 & 80 & 478.7 & 606 & 380.0 & 606 & 49.9 & 542 & 1.8 & 4051 \\
\hline & & 063595 & 3595 & 180.9 & 8 & 189.7 & 8 & 205.3 & 0 & 145.8 & 0 & 187.0 & 0 & 28.6 & 0 & 95.7 & 63 & 77.7 & 63 & 24.1 & 254 & 2.0 & 3626 \\
\hline & & 073034 & 3034 & 159.0 & 0 & 169.8 & 0 & 197.7 & 0 & 97.0 & 27 & 120.9 & 27 & 41.0 & 3 & 80.2 & 57 & 65.0 & 57 & 22.8 & 88 & 1.8 & 3085 \\
\hline & & 082588 & 2588 & 141.4 & 0 & 149.4 & 0 & 187.0 & 0 & 54.9 & 0 & 66.8 & 0 & 12.2 & 0 & 80.9 & 510 & 66.5 & 510 & 14.3 & 6 & 2.0 & 2606 \\
\hline & & 094367 & 4367 & 135.4 & 0 & 146.9 & 0 & 205.1 & 0 & 75.6 & 0 & 91.7 & 0 & 19.7 & 0 & 27.5 & 0 & 22.5 & 0 & 12.6 & 0 & 2.0 & 4405 \\
\hline & & 103407 & 3407 & 149.7 & 0 & 158.3 & 0 & 200.2 & 0 & 80.7 & 0 & 97.8 & 0 & 36.3 & 0 & 81.7 & 70 & 65.6 & 70 & 20.0 & 0 & 1.8 & 3454 \\
\hline 90 & 13 & 012252 & 2252 & 634.3 & 1754 & 698.6 & 1754 & 949.5 & 1933 & 342.5 & 3961 & 419.4 & 3961 & 171.1 & 1354 & 299.9 & 1709 & 247.2 & 1709 & 293.4 & 2134 & 3.2 & 2317 \\
\hline & & 022533 & 2533 & 743.4 & 3705 & 816.9 & 3705 & 1124.9 & 2237 & 557.1 & 5590 & 662.0 & 5590 & 554.5 & 6441 & 1520.4 & 10429 & 1321.5 & 10429 & 316.9 & 2865 & 3.4 & 2597 \\
\hline & & 032533 & 2533 & 801.7 & 3705 & 811.8 & 3705 & 1034.2 & 1991 & 545.1 & 5590 & 627.8 & 5590 & 569.6 & 5511 & 1330.5 & 10429 & 1322.3 & 10429 & 663.6 & 7412 & 3.7 & 2615 \\
\hline & & 042402 & 2402 & 401.0 & 3 & 557.2 & 3 & 854.2 & 8 & 76.2 & 7 & 88.8 & 7 & 32.4 & 64 & 71.1 & 48 & 72.2 & 48 & 30.5 & 70 & 2.9 & 2462 \\
\hline & & 052468 & 2468 & 510.6 & 50 & 570.6 & 50 & 882.1 & 60 & 128.9 & 83 & 150.9 & 83 & 42.9 & 31 & 79.8 & 102 & 80.9 & 102 & 55.5 & 110 & 3.4 & 2540 \\
\hline & & 063267 & 3267 & 776.0 & 612 & 741.2 & 612 & 969.2 & 524 & 354.4 & 752 & 437.0 & 752 & 284.1 & 1244 & 651.4 & 1934 & 659.1 & 1934 & 396.0 & 2431 & 3.6 & 3360 \\
\hline & & 072115 & 2115 & 506.8 & 7 & 537.2 & 7 & 824.3 & 7 & 61.4 & 24 & 81.5 & 24 & 13.9 & 7 & 28.4 & 7 & 28.7 & 7 & 21.4 & 29 & 3.8 & 2142 \\
\hline & & 082523 & 2523 & 528.9 & 512 & 619.7 & 512 & 852.4 & 505 & 227.2 & 810 & 280.5 & 810 & 112.2 & 848 & 564.6 & 1916 & 571.0 & 1916 & 271.3 & 2101 & 3.7 & 2603 \\
\hline & & 092844 & 2844 & 542.4 & 0 & 521.3 & 0 & 892.4 & 0 & 239.1 & 75 & 284.2 & 75 & 130.1 & 260 & 237.7 & 249 & 240.2 & 249 & 65.0 & 65 & 2.8 & 2933 \\
\hline & & 102479 & 2479 & 501.2 & 382 & 562.5 & 382 & 925.5 & 134 & 208.5 & 525 & 246.2 & 525 & 124.9 & 710 & 342.0 & 719 & 345.3 & 719 & 71.6 & 180 & 3.2 & 2558 \\
\hline 100 & & 012954 & 2954 & 1064.2 & 755 & 1095.5 & 755 & 1423.2 & 535 & 330.9 & 1843 & 402.2 & 1843 & 172.5 & 1229 & 193.9 & 951 & 196.0 & 951 & 158.8 & 1312 & 5.1 & 3026 \\
\hline & & 022775 & 2775 & 976.8 & 3657 & 957.2 & 3657 & 1544.8 & 2132 & 294.7 & 3083 & 365.4 & 3083 & 126.1 & 1896 & 669.8 & 4060 & 676.6 & 4060 & 289.6 & 4050 & 4.8 & 2867 \\
\hline & & 032617 & 2617 & 958.1 & 377 & 981.7 & 377 & 1492.2 & 501 & 223.8 & 243 & 268.6 & 243 & 73.5 & 163 & 273.9 & 262 & 277.3 & 262 & 73.5 & 260 & 3.9 & 2673 \\
\hline & & 042817 & 2817 & 2445.2 & 13148 & 2477.4 & 13148 & 3382.5 & 15874 & 2572.3 & 23009 & 3135.7 & 23009 & 2339.4 & 22812 & 3459.7 & 32884 & 3491.2 & 32884 & 1820.9 & 13910 & 4.4 & 2902 \\
\hline & & 052411 & 2411 & 791.4 & 2146 & 916.8 & 2146 & 1721.4 & 4531 & 369.0 & 4274 & 428.7 & 4274 & 259.6 & 3726 & 582.4 & 3605 & 588.0 & 3605 & 470.2 & 4668 & 4.8 & 2479 \\
\hline & & 063879 & 3879 & 1381.9 & 1356 & 1334.3 & 1356 & 1897.0 & 3089 & 796.4 & 2849 & 915.4 & 2849 & 502.2 & 2333 & 356.5 & 2036 & 359.9 & 2036 & 438.0 & 3144 & 4.5 & 3943 \\
\hline & & 072372 & 2372 & 811.6 & 5 & 718.3 & 5 & 1331.6 & 7 & 84.3 & 0 & 93.6 & 0 & 18.6 & 7 & 76.3 & 0 & 77.0 & 0 & 26.6 & 0 & 4.9 & 2403 \\
\hline & & 083281 & 3281 & 1653.4 & 4842 & 1616.1 & 4842 & 2310.0 & 9845 & 1642.1 & 15929 & 1820.1 & 15929 & 1705.6 & 14575 & 2226.1 & 12751 & 2237.5 & 12751 & 1172.4 & 8923 & 4.5 & 3374 \\
\hline & & 092993 & 2993 & 860.3 & 149 & 825.2 & 149 & 1332.7 & 83 & 155.6 & 35 & 172.1 & 35 & 42.1 & 214 & 148.1 & 163 & 149.2 & 163 & 61.4 & 586 & 4.1 & 3030 \\
\hline & & 102544 & 2544 & 1036.0 & 1813 & 1017.3 & 1813 & 1332.7 & 1778 & 333.0 & 1289 & 369.9 & 1289 & 221.0 & 2224 & 596.9 & 2537 & 508.2 & 2537 & 278.0 & 2215 & 4.7 & 2623 \\
\hline 120 & 15 & 014065 & 4065 & 988.1 & 112 & 924.9 & 112 & 1145.5 & 107 & 928.9 & 60 & 1185.4 & 60 & 283.3 & 20 & 966.4 & 63 & 756.5 & 63 & 152.4 & 21 & 12.2 & 4101 \\
\hline & & 023653 & 3653 & 521.4 & 0 & 550.5 & 0 & 858.6 & 0 & 99.7 & 0 & 123.5 & 0 & 81.9 & 66 & 61.3 & 20 & 47.6 & 20 & 29.9 & 0 & 11.6 & 3663 \\
\hline & & 033756 & 3756 & 768.8 & 714 & 774.0 & 714 & 1007.6 & 812 & 424.4 & 1757 & 527.7 & 1757 & 1139.9 & 707 & 119.1 & 329 & 98.2 & 329 & 255.1 & 538 & 11.9 & 3863 \\
\hline & & 043211 & 3211 & 1465.1 & 521 & 1390.5 & 521 & 1711.8 & 824 & 1398.2 & 768 & 1652.4 & 768 & 1433.1 & 988 & 1247.5 & 752 & 1003.6 & 752 & 2336.2 & 3686 & 11.4 & 3281 \\
\hline & & 054298 & 4298 & 7200.0 & 4428 & 7200.0 & 4079 & 4208.2 & 1551 & 1971.0 & 762 & 2303.1 & 762 & 4721.6 & 2429 & 3883.7 & 2397 & 3727.2 & 2397 & 3032.6 & 2655 & 11.3 & 4348 \\
\hline & & 064512 & 4512 & 700.2 & 80 & 758.2 & 80 & 1977.0 & 539 & 1602.9 & 577 & 1853.1 & 577 & 2173.5 & 1449 & 1680.0 & 1239 & 1680.9 & 1239 & 305.4 & 78 & 11.3 & 4588 \\
\hline & & 073463 & 3463 & 496.2 & 0 & 596.0 & 0 & 779.6 & 0 & 121.3 & 0 & 126.2 & 0 & 108.1 & 0 & 38.5 & 0 & 36.7 & 0 & 61.1 & 11 & 11.4 & 3510 \\
\hline & & 083872 & 3872 & 1294.2 & 224 & 1202.8 & 224 & 1074.1 & 0 & 866.3 & 384 & 916.4 & 384 & 274.2 & 13 & 1613.5 & 475 & 1437.2 & 475 & 149.3 & 20 & 11.6 & 3978 \\
\hline & & 094176 & 4176 & 1600.7 & 1132 & 1582.2 & 1132 & 2054.9 & 784 & 1059.4 & 254 & 1072.5 & 254 & 1114.6 & 726 & 1962.4 & 1606 & 1974.5 & 1606 & 1110.1 & 2095 & 11.2 & 4294 \\
\hline & & 103880 & 3880 & 2843.8 & 7347 & 2938.3 & 7347 & 2746.8 & 4309 & 868.8 & 958 & 877.8 & 958 & 2851.7 & 6484 & 1536.7 & 1768 & 1550.1 & 1768 & 1876.3 & 6135 & 11.7 & 3939 \\
\hline 150 & 15 & 018219 & 8219 & 7200.0 & 402 & 7200.0 & 524 & 7200.0 & 900 & 7200.0 & 1035 & 7200.0 & 1273 & 7200.0 & 544 & 7200.0 & 1798 & 7200.0 & 2055 & 7027.8 & 3336 & 24.0 & 8305 \\
\hline & & 026737.7 & 6742 & 7200.0 & 462 & 7200.0 & 550 & 7200.0 & 920 & 7200.0 & 527 & 7200.0 & 521 & 7200.0 & 499 & 7200.0 & 1500 & 7200.0 & 1497 & 7200.0 & 2709 & 24.3 & 6854 \\
\hline & & 034655 & 4655 & 858.0 & 0 & 922.4 & 0 & 1177.0 & 0 & 279.3 & 0 & 284.5 & 0 & 213.5 & 0 & 51.0 & 0 & 51.0 & 0 & 68.3 & 40 & 25.2 & 4754 \\
\hline & & 047303 & 7303 & 7200.0 & 3138 & 7200.0 & 4252 & 5195.7 & 2128 & 7200.0 & 2119 & 7200.0 & 2052 & 7200.0 & 620 & 7200.0 & 493 & 7200.0 & 487 & 7200.0 & 1820 & 23.5 & 7365 \\
\hline & & 056563 & 6563 & 3065.4 & 3854 & 2665.3 & 3854 & 7200.0 & 8126 & 7200.0 & 8698 & 7200.0 & 8616 & 7200.0 & 7297 & 4092.0 & 28055 & 3761.3 & 28055 & 2258.5 & 3629 & 24.5 & 6623 \\
\hline & & 066348 & 6359 & 7200.0 & 557 & 7200.0 & 795 & 7200.0 & 965 & 7200.0 & 422 & 7200.0 & 420 & 7200.0 & 693 & 7200.0 & 402 & 7200.0 & 662 & 7200.0 & 1664 & 24.2 & 6434 \\
\hline & & 076343 & 6343 & 5145.4 & 1673 & 5503.4 & 1673 & 5456.2 & 1427 & 5076.3 & 1714 & 5174.9 & 1714 & 5713.0 & 1909 & 5088.5 & 1932 & 4914.7 & 1932 & 7006.9 & 1928 & 23.6 & 6452 \\
\hline & & 087940 & 7940 & 7200.0 & 85081 & 7200.0 & 74811 & 5364.3 & 24680 & 7200.0 & 4348 & 7200.0 & 3871 & 7200.0 & 63629 & 7200.0 & 105292 & 7200.0 & 103727 & 7035.6 & 45537 & 23.7 & 8002 \\
\hline & & 098242 & 8242 & 3969.9 & 6257 & 4086.0 & 6257 & 2137.7 & 961 & 4265.1 & 10150 & 4815.8 & 10150 & 2530.9 & 1087 & 4510.6 & 11856 & 4533.8 & 11856 & 3414.4 & 10018 & 23.4 & 8284 \\
\hline & & 106012.9 & 6016 & 7200.0 & 3946 & 7200.0 & 3559 & 7200.0 & 5953 & 7200.0 & 2637 & 7200.0 & 2274 & 7200.0 & 2874 & 7200.0 & 2956 & 7200.0 & 2980 & 7200.0 & 3137 & 23.2 & 6057 \\
\hline
\end{tabular}

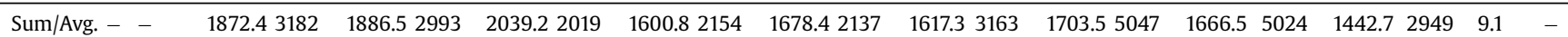


Table A.16

Detailed results for the new benchmark instances.

\begin{tabular}{|c|c|c|c|c|c|c|c|c|c|c|c|c|c|c|}
\hline \multirow[t]{3}{*}{$n$} & \multirow[t]{3}{*}{$m$} & \multirow[t]{3}{*}{ id } & \multirow[t]{3}{*}{$\mathrm{lb}$} & \multirow[t]{3}{*}{ ub } & \multicolumn{4}{|c|}{ Time-indexed } & \multicolumn{4}{|c|}{ Arc-flow } & \multicolumn{2}{|l|}{$\mathrm{MH}$} \\
\hline & & & & & \multicolumn{2}{|l|}{$\mathrm{TI}_{+}$} & \multicolumn{2}{|l|}{$\mathrm{TI}_{+}^{\mathrm{rc}}$} & \multicolumn{2}{|l|}{$\mathrm{AF}_{+}$} & \multicolumn{2}{|l|}{$\mathrm{AF}_{+}^{\mathrm{rc}}$} & \multirow[b]{2}{*}{$\mathrm{t}(\mathrm{s})$} & \multirow[b]{2}{*}{$\mathrm{ub}$} \\
\hline & & & & & $\mathrm{t}(\mathrm{s})$ & nd & $\mathrm{t}(\mathrm{s})$ & nd & $\mathrm{t}(\mathrm{s})$ & nd & $\mathrm{t}(\mathrm{s})$ & nd & & \\
\hline \multirow[t]{10}{*}{200} & \multirow[t]{10}{*}{15} & 01 & 12604 & 12609 & tlim & 98 & tlim & 396 & tlim & 68 & tlim & 113 & 67.6 & 12709 \\
\hline & & 02 & 10319 & 10319 & 6301.8 & 116 & tlim & 177 & 3620.8 & 114 & 1971.0 & 41 & 60.0 & 10407 \\
\hline & & 03 & 11296 & 11355 & tlim & 47 & tlim & 318 & tlim & 108 & tlim & 218 & 37.1 & 11558 \\
\hline & & 04 & 15441 & 15441 & tlim & 107 & tlim & 300 & 6880.6 & 343 & 3671.1 & 1499 & 34.7 & 15647 \\
\hline & & 05 & 18166 & 18352 & tlim & 11 & tlim & 67 & tlim & 17 & tlim & 35 & 35.7 & 18352 \\
\hline & & 06 & 16869 & 16869 & 6836.4 & 567 & 6491.5 & 577 & 1015.5 & 531 & 1612.8 & 224 & 36.9 & 16961 \\
\hline & & 07 & 13025 & 13226 & tlim & 37 & tlim & 66 & tlim & 81 & tlim & 78 & 35.5 & 13226 \\
\hline & & 08 & 14182 & 14259 & tlim & 7 & tlim & 9 & tlim & 48 & $\operatorname{tlim}$ & 58 & 36.1 & 14537 \\
\hline & & 09 & 18118 & 18118 & tlim & 100 & 1946.9 & 105 & 3879.7 & 132 & 4987.1 & 657 & 35.5 & 18198 \\
\hline & & 10 & 17102 & 17118 & tlim & 19 & tlim & 39 & tlim & 68 & tlim & 203 & 35.0 & 17263 \\
\hline \multirow[t]{10}{*}{250} & \multirow[t]{10}{*}{20} & 01 & 15633 & 15769 & tlim & 7 & tlim & 27 & tlim & 13 & tlim & 34 & 78.0 & 15769 \\
\hline & & 02 & 15776 & 15915 & tlim & 5 & tlim & 20 & tlim & 24 & tlim & 55 & 84.0 & 15915 \\
\hline & & 03 & 16519 & 16606 & tlim & 4 & tlim & 8 & tlim & 15 & tlim & 28 & 77.9 & 16724 \\
\hline & & 04 & 16423 & 16481 & tlim & 17 & tlim & 46 & tlim & 14 & tlim & 33 & 83.0 & 16509 \\
\hline & & 05 & 15661 & 15837 & tlim & 1 & tlim & 2 & tlim & 14 & tlim & 34 & 77.3 & 15837 \\
\hline & & 06 & 20060 & 20060 & tlim & 0 & tlim & 4 & 5180.6 & 10 & tlim & 19 & 82.6 & 20193 \\
\hline & & 07 & 14284 & 14362 & tlim & 0 & tlim & 25 & tlim & 21 & tlim & 38 & 84.1 & 14514 \\
\hline & & 08 & 16305 & 16383 & tlim & 0 & tlim & 12 & tlim & 10 & tlim & 20 & 79.4 & 16498 \\
\hline & & 09 & 15864 & 15917 & tlim & 0 & tlim & 2 & tlim & 16 & tlim & 26 & 82.5 & 16121 \\
\hline & & 10 & 16283 & 16371 & tlim & 0 & tlim & 24 & tlim & 10 & tlim & 21 & 81.0 & 16428 \\
\hline Avg. & & & - & - & 7136.9 & 57 & 6901.9 & 111 & 6428.9 & 83 & 6372.1 & 172 & 61.2 & - \\
\hline
\end{tabular}

\section{References}

Agra, A., \& Oliveira, M. (2018). MIP approaches for the integrated berth allocation and quay crane assignment and scheduling problem. European Journal of Operational Research, 264(1), 138-148.

Bierwirth, C., \& Meisel, F. (2010). A survey of berth allocation and quay crane scheduling problems in container terminals. European Journal of Operational Research, 202(3), 615-627.

Bierwirth, C., \& Meisel, F. (2015). A follow-up survey of berth allocation and quay crane scheduling problems in container terminals. European Journal of Operational Research, 244(3), 675-689.

Brooks, M. R., \& Faust, P. (2018). 50 years of review of maritime transport, 1968-2018: reflecting on the past, exploring the future. Technical Report, UNCTAD.

Buhrkal, K., Zuglian, S., Ropke, S., Larsen, J., \& Lusby, R. (2011). Models for the discrete berth allocation problem: A computational comparison. Transportation Research Part E, 47(4), 461-473.

Choi, H. R., Kim, H. S., Park, B. J., Park, N.-K., \& Lee, S. W. (2003). An ERP approach for container terminal operating systems. Maritime Policy \& Management, 30(3), $197-210$.

Christensen, C., \& Holst, C. (2008). Berth allocation in container terminals. Master's thesis. Technical University of Denmark.

Cordeau, J.-F., Gaudioso, M., Laporte, G., \& Moccia, L. (2007). The service allocation problem at the Gioia Tauro maritime terminal. European Journal of Operational Research, 176(2), 1167-1184.

Cordeau, J.-F., Laporte, G., Legato, P., \& Moccia, L. (2005). Models and tabu search heuristics for the berth-allocation problem. Transportation Science, 39(4), $526-538$.

Delorme, M., Iori, M., \& Martello, S. (2016). Bin packing and cutting stock problems: Mathematical models and exact algorithms. European Journal of Operational Research, 255(1), 1-20.

De Oliveira, R. M., Mauri, G. R., \& Lorena, L. A. N. (2012). Clustering search for the berth allocation problem. Expert Systems with Applications, 39(5), 5499-5505.

Fischetti, M., \& Monaci, M. (2014). Exploiting erraticism in search. Operations Research, 62(1), 114-122.

Frojan, P., Correcher, J. F., Álvarez-Valdés, R., Koulouris, G., \& Tamarit, J. M. (2015). The continuous berth allocation problem in a container terminal with multiple quays. Expert Systems with Applications, 42(21), 7356-7366.

Giallombardo, G., Moccia, L., Salani, M., \& Vacca, I. (2010). Modeling and solving the tactical berth allocation problem. Transportation Research Part B: Methodological, 44(2), 232-245.

Heilig, L., \& Voß, S. (2018). Port-centric information management in smart ports: A framework and categorization. In H. Geerlings, B. Kuipers, \& R. Zuidwijk (Eds.), Ports and Networks: Strategies, Operations and Perspectives (pp. 236-250). Routledge, Oxon and New York.

Imai, A., Nishimura, E., \& Papadimitriou, S. (2001). The dynamic berth allocation problem for a container port. Transportation Research Part B: Methodological, 35(4), 401-417.

Imai, A., Yamakawa, Y., \& Huang, K. (2014). The strategic berth template problem. Transportation Research Part E: Logistics and Transportation Review, 72, 77-100.
Kramer, A., Dell'Amico, M., \& Iori, M. (2019). Enhanced arc-flow formulations to minimize weighted completion time on identical parallel machines, European Journal of Operational Research, 275(1), 67-79.

Lalla-Ruiz, E. (2016). Intelligent management of seaside logistic operations at maritime container terminals. 40R, 2(15), 217-218.

Lalla-Ruiz, E., Melián-Batista, B., \& Moreno-Vega, J. M. (2012). Artificial intelligence hybrid heuristic based on tabu search for the dynamic berth allocation problem. Engineering Applications of Artificial Intelligence, 25(6), 1132-1141.

Lalla-Ruiz, E., Melián-Batista, B., \& Moreno-Vega, J. M. (2016). A cooperative search for berth scheduling. The Knowledge Engineering Review, 31(5), 498-507.

Lalla-Ruiz, E., \& Voß, S. (2016a). Improving solver performance through redundancy. Journal of Systems Science and Systems Engineering, 25(3), 303-325.

Lalla-Ruiz, E., \& Voß, S. (2016b). POPMUSIC as a matheuristic for the berth allocation problem. Annals of Mathematics and Artificial Intelligence, 76(1), 173-189.

Lodi, A., \& Tramontani, A. (2013). Performance variability in mixed-integer programming. In $\mathrm{H}$. Topaloglu (Ed.), TutORials in operations research: Theory driven by influential applications (pp. 1-12). INFORMS.

Mauri, G. R., Ribeiro, G. M., Lorena, L. A. N., \& Laporte, G. (2016). An adaptive large neighborhood search for the discrete and continuous berth allocation problem. Computers \& Operations Research, 70, 140-154.

Nishi, T., Okura, T., Lalla-Ruiz, E., \& Voß, S. (2016). A dynamic programming-based matheuristic for the dynamic berth allocation problem. Annals of Operations Research, 1-20, online available.

Notteboom, T. (2006). The time factor in liner shipping services. Maritime Economics \& Logistics, 8(1), 19-39.

Şahin, C., \& Kuvvetli, Y. (2016). Differential evolution based meta-heuristic algorithm for dynamic continuous berth allocation problem. Applied Mathematical Modelling, 40(23-24), 1339-1351.

Savelsbergh, M. W. (1994). Preprocessing and probing techniques for mixed integer programming problems. ORSA Journal on Computing, 6(4), 445-454.

Sousa, J. P., \& Wolsey, L. A. (1992). A time indexed formulation of non-preemptive single machine scheduling problems. Mathematical Programming, 54(1), 353-367.

Stahlbock, R., \& Voß, S. (2008). Vehicle routing problems and container terminal operations-an update of research. In The vehicle routing problem: Latest advances and new challenges (pp. 551-589). Springer.

Steenken, D., Voß, S., \& Stahlbock, R. (2004). Container terminal operations and operations research - a classification and literature review. OR Spectrum, 26(1), 3-49.

Ting, C.-J., Wu, K.-C., \& Chou, H. (2014). Particle swarm optimization algorithm for the berth allocation problem. Expert Systems with Applications, 41(4), 1543-1550.

Valério de Carvalho, J. (2002). LP models for bin packing and cutting stock problems. European Journal of Operational Research, 141(2), 253-273.

Valério de Carvalho, J. (1999). Exact solution of bin-packing problems using column generation and branch-and-bound. Annals of Operations Research, 86(0), 629-659.

Xu, D., Li, C.-L., \& Leung, J. Y.-T. (2012). Berth allocation with time-dependent physical limitations on vessels. European Journal of Operational Research, 216(1) $47-56$. 